\title{
On Shape Optimization for an Evolution Coupled System
}

\author{
G. Leugering • A.A. Novotny • G. Perla Menzala • \\ J. Sokołowski
}

Published online: 21 September 2011

(C) The Author(s) 2011. This article is published with open access at Springerlink.com

\begin{abstract}
A shape optimization problem in three spatial dimensions for an elastodynamic piezoelectric body coupled to an acoustic chamber is introduced. Wellposedness of the problem is established and first order necessary optimality conditions are derived in the framework of the boundary variation technique. In particular, the existence of the shape gradient for an integral shape functional is ob-
\end{abstract}

Author G.L. acknowledges support by the DFG-Cluster of Excellence: Engineering of Advanced Materials (grant).

Author G.P.M acknowledges support by the Brazilian Agency CNPq under grant 301134/2009-0. Authors A.A.N. and J.S. acknowledge support by the Brazilian-French research programme CAPES/COFECUB under grant 604/08 between LNCC (Petrópolis) and IECN (Nancy). Author J.S. acknowledge the support of ANR project GAOS.

G. Leugering

Department of Mathematics, University of Erlangen Nuremberg, Martenstr. 3, 91058 Erlangen, Germany

e-mail: leugering@am.uni-erlangen.de

A.A. Novotny · G. Perla Menzala

Laboratório Nacional de Computação Científica LNCC/MCT, Coordenação de Matemática Aplicada e Computacional, Av. Getúlio Vargas 333, 25651-075 Petrópolis, RJ, Brasil
A.A. Novotny
e-mail: novotny@lncc.br
G. Perla Menzala
e-mail: perla@lncc.br

J. Sokołowski $(\bowtie)$

Institut Elie Cartan, UMR 7502 (Nancy Université, CNRS, INRIA), Laboratoire de Mathématiques, Université Henri Poincaré Nancy I, B.P. 239, 54506 Vandoeuvre-lès-Nancy Cedex, France e-mail: Jan.Sokolowski@iecn.u-nancy.fr

J. Sokołowski

Systems Research Institute of the Polish Academy of Sciences, ul. Newelska 6, 01-447 Warszawa, Poland 
tained, as well as its regularity, sufficient for applications e.g. in modern loudspeaker technologies. The shape gradients are given by functions supported on the moving boundaries. The paper extends results obtained by the authors in (Math. Methods Appl. Sci. 33(17):2118-2131, 2010) where a similar problem was treated without acoustic coupling.

Keywords Piezoelectricity · Electromechanical interaction · Shape sensitivity analysis

\section{Introduction}

Shape optimization for coupled models is an emerging field of research required for applications in modern key-technologies. In the present paper a model for interactions between elastic, piezo-electric and acoustic fields with non stationary partial differential equations is proposed and analyzed. The geometrical domain is decomposed into regions with different physical properties, and the sub-domains are coupled by means of appropriate transmission conditions for the equations under considerations. The problem is chosen in such a way, that the results can be applied for a broad class of models, with the appropriate modifications, if necessary. The configuration is viewed e.g. as a loudspeaker in an acoustic chamber. The question asked in applications concerning loudspeakers, beepers or energy harvesters is about the shape and the topology of the material components involved. See [13-16] for the original engineering problem formulation along with topology optimization results based on the classical SIMP method. Indeed, a main objective is e.g. to maximize the acoustic pressure in the chamber by choosing appropriately shaped elasto-piezo-systems. However, in these articles the problem was concerned with optimizing the topology of the piezo-patches only. Moreover, only a time-harmonic solution was considered. Time dependent piezo-electric coupled systems have been investigated in the literature before, e.g. in $[10,11]$. Multilayered piezo-actuator devices have been studied e.g. in [5]. In [9], the dynamic problem without acoustic coupling was first studied with respect to well-posedness and shape-sensitivity analysis. See the references in [9] for further information about the literature in this context. In this paper the same authors consider the fully coupled dynamic system involving also the acoustic chamber. For the mathematical theory concerning the evolution problems the reader may refer to e.g., [8].

In order to avoid additional difficulties with respect to geometrical singularities, and in order to have a simpler presentation of the results, we decide to use a layered system as in Fig. 1.

Topological sensitivity analysis is not performed in the paper, however we can refer to the related papers which include the topological derivatives for the stationary models. The shape and topological sensitivity analysis of partial differential equations is an efficient tool in numerical solution of optimum design problems for distributed parameter systems. We are interested in shape sensitivity analysis in three spatial dimensions of the complete model of the interaction between elastic, piezoelectric and acoustic fields. There is a difference between stationary problems and evolution problems in this respect. To be more precise, the difference concerns the singular 
Fig. 1 Layered domain represented by $\Omega$

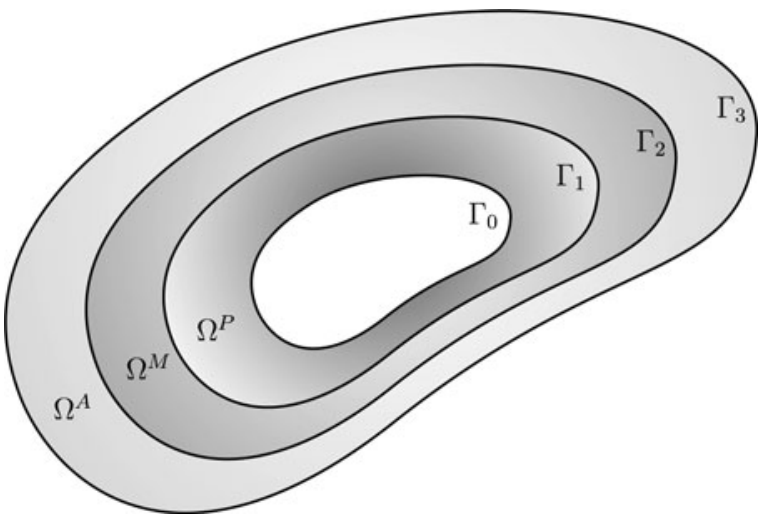

domain perturbations, e.g. the analysis of the influence of nucleation of small voids on the solutions of the mathematical model. Such an analysis can be performed in the framework compound and matched asymptotic expansions for stationary models, and it is unknown in the case of full evolution model. The asymptotic analysis is not, however, our principal activity in the present paper, we refer the reader to [2] for some results in this direction for piezoelectric elastic bodies.

The techniques of boundary variations, which we employ in the paper, is the speed method. By this method, material and shape derivatives are determined for the partial differential equations involved, and the Hadamard structure theorem for shape gradients is used in order to identify the boundary density function of the shape gradient which, in turn, can be used in numerical methods for shape optimization.

This means that the first part of our analysis in the framework of shape sensitivity analysis is devoted to the so-called material derivatives of solutions to the boundary value problems in the stationary case or to the evolution initial-boundary value problems in the evolution case. The analysis which leads to the material derivatives is usually performed in the fixed domain setting by an application of the implicit function theorem. To this end transport mapping for the family of admissible domains is constructed, and by construction the mapping is a diffeomorphism in three spatial dimensions between admissible domains. We need some regularity of domains and of the mappings to assure all necessary properties of the diffeomorphism.

In optimum design of elastic structures the topological derivatives can be determined by asymptotic analysis with respect to the small parameter which governs perturbations of coefficients in a regular case or singular domain perturbations in limit cases of small voids and/or rigid inclusions. We point out that for evolution problems that case of singular domain perturbations is still out of the reach, however regular perturbations in coefficients make no additional difficulties compared with the shape sensitivity analysis.

In the paper the shape gradient (5.48) is obtained for shape functional (2.8) defined for the model introduced in Sect. 2.1. We need the expression of the shape gradient to be given by a function, for the purposes of numerical methods of shape optimization. Therefore, the regularity issue we address in the paper can be described as follows. Under minimal regularity assumptions for the model and for the shape functional, determine the expressions for Eshelby tensors (5.36) and (5.37) in such a way, that 
the traces of tensors on moving boundaries are given by functions. Therefore, the shape gradient is given by a function and the levelset methods of shape optimization can be applied in order to solve numerically the associated optimization problems.

\section{The Problem Formulation}

Let us consider an open bounded domain $\Omega$ of $\mathbb{R}^{3}$ with smooth boundary $\partial \Omega$. We assume that $\Omega$ has the form $\Omega=\mathcal{D} \backslash \overline{\mathcal{D}_{0}}$, where $\mathcal{D}$ and $\mathcal{D}_{0}$ are open bounded domains with $\overline{\mathcal{D}_{0}} \subset \mathcal{D}$ and $\bar{\Omega}$ denotes the closure of $\Omega$. In addition, let $\mathcal{B}_{i}$, with $i=0,1,2,3$, be open subsets with smooth boundary $\Gamma_{i}$, such that, for $j=0,1,2, \overline{\mathcal{B}_{j}} \subset \mathcal{B}_{j+1}$, with $\mathcal{B}_{0}=\mathcal{D}_{0}$ and $\mathcal{B}_{3}=\mathcal{D}$. We set $\Omega^{P}=\mathcal{B}_{1} \backslash \overline{\mathcal{B}_{0}}, \Omega^{M}=\mathcal{B}_{2} \backslash \overline{\mathcal{B}_{1}}$ and $\Omega^{A}=\mathcal{B}_{3} \backslash \overline{\mathcal{B}_{2}}$. In summary, as shown in Fig. 1, the mutually disjoints open domains $\Omega^{P}, \Omega^{M}, \Omega^{A}$ have boundaries $\partial \Omega^{P}=\Gamma_{0} \cup \Gamma_{1}, \partial \Omega^{M}=\Gamma_{1} \cup \Gamma_{2}$ and $\partial \Omega^{A}=\Gamma_{2} \cup \Gamma_{3}$, respectively. We remark that the order of domains can be chosen in reverse order such that the acoustic part is inside and represents an acoustic chamber.

According to our above motivation, $\Omega^{M}$ and $\Omega^{P}$ represent the regions where mechanical and piezoelectric devices are located, respectively, and $\Omega^{A}$ represents the acoustic chamber.

\subsection{The Model}

We are interested in the following system

$$
\begin{cases}\frac{1}{c^{2}} \varphi_{t t}-\Delta \varphi=f & \text { in } \Omega^{A} \times(0, T), \\ w_{t t}-\operatorname{div} S=g & \text { in } \Omega^{M} \times(0, T), \\ u_{t t}-\operatorname{div} \sigma=h & \text { in } \Omega^{P} \times(0, T), \\ -\operatorname{div} \psi=0 & \end{cases}
$$

where the first equation describes the acoustic wave propagation, the second one is the linear elasticity system and the last coupled system represents the electromechanical interaction phenomenon. The equations are coupled at layers $\Gamma_{j}(j=1,2)$. In particular, $\varphi$ is the acoustic potential scalar field, $S$ is the mechanical stress tensor, $\sigma$ is the electromechanical stress tensor and $\psi$ the electrical displacement field. The constitutive laws describing the elastic behavior and piezoelectric effects, both in the linearized case of small mechanical deformations and electric fields, are

$$
\left\{\begin{array}{l}
S(w)=A \varepsilon(w), \\
\sigma(u, q)=C \varepsilon(u)-P e(q), \\
\psi(u, q)=P^{\top} \varepsilon(u)+\operatorname{De}(q),
\end{array}\right.
$$

where $w=w(x, t)$ and $u=u(x, t)$ are the mechanical and electromechanical displacements, respectively, and $q=q(x, t)$ is the electric potential. In addition, $A$ and $C$ are the elasticity fourth-order tensors respectively associated to the elastic and electromechanical parts, $P$ the piezoelectric coupling third-order tensor and $D$ the 
dielectric second-order tensor. As usual $A, C$ and $D$ satisfy the symmetry conditions $A_{i j k l}=A_{j i k l}=A_{k l i j}, C_{i j k l}=C_{j i k l}=C_{k l i j}$ and $D_{i j}=D_{j i}$, whereas $P$ satisfies $P_{i j k}=P_{j i k}$. Furthermore, there exist nonnegative constants $a_{0}, c_{0}$ and $d_{0}$ such that

$$
A_{i j k l} X_{i j} X_{k l} \geq a_{0} X_{i j}^{2}, \quad C_{i j k l} Y_{i j} Y_{k l} \geq c_{0} Y_{i j}^{2}, \quad D_{i j} z_{i} z_{j} \geq d_{0} z_{i}^{2},
$$

where Einstein's summation convention is used. It is assumed for simplicity that all constitutive tensors are piecewise constant, i.e., constant in each layer. The mechanical strain tensors $\varepsilon(u), \varepsilon(w)$ and the electric vector field $e(q)$ are given by

$$
\begin{aligned}
& \varepsilon(u)=\nabla^{s} u:=\frac{1}{2}\left(\nabla u+\nabla u^{\top}\right), \\
& \varepsilon(w)=\nabla^{s} w:=\frac{1}{2}\left(\nabla w+\nabla w^{\top}\right) \text { and } e(q)=-\nabla q .
\end{aligned}
$$

We complement the system (2.1) with the following initial conditions

$$
\begin{cases}\varphi(x, 0)=\varphi_{0}(x), & \varphi_{t}(x, 0)=\varphi_{1}(x) \\ w(x, 0)=w_{0}(x), & w_{t}(x, 0)=w_{1}(x) \\ u(x, 0)=u_{0}(x), & u_{t}(x, 0)=u_{1}(x)\end{cases}
$$

and boundary conditions of the form

$$
\left\{\begin{array}{l}
\psi \cdot n=0 \\
u=0
\end{array} \quad \text { on } \Gamma_{0} \times(0, T), \quad \frac{\partial \varphi}{\partial n}=-\frac{1}{c} \varphi_{t} \quad \text { on } \Gamma_{3} \times(0, T),\right.
$$

where $n$ is the outward unit normal vector pointing toward the exterior of $\Omega$. Finally, we consider the following transmission conditions

$$
\left\{\begin{array} { l } 
{ u = w } \\
{ \sigma n = S n } \\
{ q = q ^ { P } }
\end{array} \quad \text { on } \Gamma _ { 1 } \times ( 0 , T ) \quad \text { and } \quad \left\{\begin{array}{l}
w_{t} \cdot n=-\frac{\partial \varphi}{\partial n} \\
S n=-\varphi_{t} n
\end{array} \quad \text { on } \Gamma_{2} \times(0, T)\right.\right.
$$

where $n=n^{(i)}=-n^{(i-1)}$ is the unit normal vector pointing toward the exterior of $\mathcal{B}_{i}$. We also assume the compatibility condition $q^{P}(x, 0)=q_{t}^{P}(x, 0)=0$.

\subsection{Shape Functional}

We consider a shape functional of the form

$$
\mathcal{J}_{\Omega}\left(\varphi_{t}, w\right)=\int_{0}^{T} J_{\Omega}\left(\varphi_{t}, w\right)
$$

with $J_{\Omega}\left(\varphi_{t}, w\right)$ defined as

$$
J_{\Omega}\left(\varphi_{t}, w\right):=\alpha \frac{1}{2} \int_{\Omega^{A}}\left(\varphi_{t}-p^{\star}\right)^{2}-\beta \int_{\Omega^{M}}(\operatorname{div}(w) \eta+w \cdot \nabla \eta),
$$


where $p^{\star}$ is a target acoustic pressure, $\eta$ is an arbitrary scalar function, $\alpha=1-\beta$ and $\beta \in[0,1]$. By taking $\left.\eta\right|_{\Gamma_{1}}=0$ and $\left.\eta\right|_{\Gamma_{2}}=1$, we have

$$
J_{\Omega}\left(\varphi_{t}, w\right)=\alpha \frac{1}{2} \int_{\Omega^{A}}\left(\varphi_{t}-p^{\star}\right)^{2}-\beta \int_{\Gamma_{2}} w \cdot n,
$$

where $w \cdot n$ is the normal component of the mechanical displacement on the interface $\Gamma_{2}$ between the acoustic chamber and the mechanical device, respectively represented by $\Omega^{A}$ and $\Omega^{M}$. It means that we want to maximize the mechanical displacement and the acoustic pressure by taking $p^{\star}$ large enough.

\section{State Equations}

In this section the existence and the regularity of weak solutions to the model of coupled equations in multilayered domain is established by Theorem 1 . The same results are valid for other coupled systems introduced in the paper, including the material and shape derivatives as well as the adjoint state equations.

\subsection{Weak Solutions}

In order to derive a weak formulation of the piezoelectric problem (2.1)-(2.6) we introduce the following bilinear forms

$$
\begin{aligned}
a_{A}(\varphi, \varphi) & :=\langle\nabla \varphi, \nabla \varphi\rangle_{\Omega^{A}}, \\
a_{M}(w, w) & :=\left\langle A \nabla^{s} w, \nabla^{s} w\right\rangle_{\Omega^{M}}, \\
a_{M M}(u, u) & :=\left\langle C \nabla^{s} u, \nabla^{s} u\right\rangle_{\Omega^{P}}, \\
a_{E E}(q, q) & :=\langle D \nabla q, \nabla q\rangle_{\Omega^{P}}, \\
a_{M E}(u, q) & :=\left\langle P^{\top} \nabla^{s} u, \nabla q\right\rangle_{\Omega^{P}}, \\
a_{E M}(q, u) & :=\left\langle P \nabla q, \nabla^{s} u\right\rangle_{\Omega^{P}},
\end{aligned}
$$

and spaces

$$
\begin{aligned}
& \mathcal{W}_{A}=H^{1}\left(\Omega^{A}\right), \quad \mathcal{W}_{M}=\left[H^{1}\left(\Omega^{M}\right)\right]^{3}, \\
& \mathcal{W}_{P}=\left[H^{1}\left(\Omega^{P}\right)\right]^{3}, \quad \mathcal{W}_{E}=H^{1}\left(\Omega^{P}\right),
\end{aligned}
$$

as well as

$$
\begin{gathered}
\mathcal{W}=\left\{(\varphi, w, u, q)(t) \in \mathcal{W}_{A} \times \mathcal{W}_{M} \times \mathcal{W}_{P} \times \mathcal{W}_{E}:\right. \\
\left.\quad u=0 \text { on } \Gamma_{0}, w=u \text { on } \Gamma_{1} \text { and } q=q^{P}(t) \text { on } \Gamma_{1}, \text { for each } t \in(0, T)\right\} \\
\tilde{\mathcal{W}}=\left\{(\widetilde{\varphi}, \widetilde{w}, \widetilde{u}, \widetilde{q}) \in \mathcal{W}_{A} \times \mathcal{W}_{M} \times \mathcal{W}_{P} \times \mathcal{W}_{E}:\right. \\
\left.\tilde{u}=0 \text { on } \Gamma_{0}, \tilde{w}=\tilde{u} \text { on } \Gamma_{1} \text { and } \tilde{q}=0 \text { on } \Gamma_{1}\right\}
\end{gathered}
$$


Then the weak formulation of (2.1)-(2.6) is obtained by multiplying the equations with test functions $(\widetilde{\varphi}, \widetilde{w}, \widetilde{u}, \widetilde{q}) \in \tilde{\mathcal{W}}(\Omega)$, respectively, followed by integration by parts. It reads: for each $t \in(0, T)$ and any $(\widetilde{\varphi}, \widetilde{w}, \widetilde{u}, \widetilde{q}) \in \mathcal{W}(\Omega)$, find the acoustic potential $\varphi$, the mechanical displacement $w$, the electromechanical displacement $u$ and the electric potential $q$, with $(\varphi, w, u, q) \in \mathcal{W}$, such that

$$
\left\{\begin{array}{l}
\frac{1}{c^{2}}\left\langle\varphi_{t t}(t), \widetilde{\varphi}\right\rangle_{\Omega^{A}}+a_{A}(\varphi(t), \widetilde{\varphi})-\left\langle w_{t}(t) \cdot n, \widetilde{\varphi}\right\rangle_{\Gamma_{2}}+\frac{1}{c}\left\langle\varphi_{t}(t), \widetilde{\varphi}\right\rangle_{\Gamma_{3}} \\
\quad+\left\langle w_{t t}(t), \widetilde{w}\right\rangle_{\Omega^{M}}+a_{M}(w(t), \widetilde{w})+\left\langle\varphi_{t}(t), \widetilde{w} \cdot n\right\rangle_{\Gamma_{2}} \\
\quad+\left\langle u_{t t}(t), \widetilde{u}\right\rangle_{\Omega^{P}}+a_{M M}(u(t), \widetilde{u})+a_{E M}(q(t), \widetilde{u}) \\
\quad+a_{E E}(q(t), \widetilde{q})-a_{M E}(u(t), \widetilde{q})=0 .
\end{array}\right.
$$

In order to put this into a more convenient format, we introduce the variable $W:=$ $(\varphi, w, u, q)$ and the bilinear forms

$$
\begin{aligned}
\mathcal{A}(W, \widetilde{W}):= & a_{A}(\varphi, \widetilde{\varphi})+a_{M}(w, \widetilde{w})+a_{M M}(u, \widetilde{u})+a_{E E}(q, \widetilde{q})+a_{E M}(q, \widetilde{u}) \\
& -a_{M E}(u, \widetilde{q}), \\
\mathcal{B}(W, \widetilde{W}):= & -\langle w \cdot n, \widetilde{\varphi}\rangle_{\Gamma_{2}}+\langle\varphi, \widetilde{w} \cdot n\rangle_{\Gamma_{2}}+\frac{1}{c}\langle\varphi, \widetilde{\varphi}\rangle_{\Gamma_{3}},
\end{aligned}
$$

where the symbol $\langle\cdot, \cdot\rangle_{K}$ denotes the usual inner product for elements of functional spaces defined in a domain $K$. Notice that

$$
\begin{aligned}
& \mathcal{A}(W, W)=a_{A}(\varphi, \varphi)+a_{M}(w, w)+a_{M M}(u, u)+a_{E E}(q, q), \\
& \mathcal{B}(W, W)=\frac{1}{c}\langle\varphi, \varphi\rangle_{\Gamma_{3}} .
\end{aligned}
$$

The space $\widetilde{\mathcal{W}}$ can be seen as the form-domain of $\mathcal{A}(\cdot, \cdot)$. The weak system (3.4) can be rewritten as

$$
\left\langle\mathcal{M} W_{t t}, \widetilde{W}\right\rangle_{\Omega}+\mathcal{B}\left(W_{t}, \widetilde{W}\right)+\mathcal{A}(W, \widetilde{W})=0, \quad \forall W \in \widetilde{W},
$$

where $\mathcal{M}=\operatorname{diag}\left(\frac{1}{c^{2}} I, I, I, 0\right)$. Still, (3.7) is not a standard vectorial dissipative wave equation in weak form, the mass matrix-operator $\mathcal{M}$ is singular. Therefore, a proof of well-posedness seems to be at order.

Theorem 1 Given $f \in L^{2}\left(0, T ; L^{2}\left(\Omega^{A}\right)\right), g \in L^{2}\left(0, T ;\left[L^{2}\left(\Omega^{M}\right)\right]^{3}\right), h \in L^{2}(0, T$; $\left.\left[L^{2}\left(\Omega^{P}\right)\right]^{3}\right),\left(\varphi_{0}, w_{0}, u_{0}, 0\right) \in \tilde{\mathcal{W}},\left(\varphi_{1}, w_{1}, u_{1}, 0\right) \in L^{2}\left(\Omega^{A}\right) \times\left[L^{2}\left(\Omega^{M}\right)\right]^{3} \times\left[L^{2}\left(\Omega^{P}\right)\right]^{3}$ $\times\{0\}$ and compatibility condition $q^{P}(x, 0)=q_{t}^{P}(x, 0)=0$, then, there exists $a$ unique weak solution to (3.4) belonging to the class

$$
\begin{array}{ll}
\varphi \in L^{\infty}\left(0, T ; H^{1}\left(\Omega^{A}\right)\right), & \varphi_{t} \in L^{\infty}\left(0, T ; L^{2}\left(\Omega^{A}\right)\right), \\
w \in L^{\infty}\left(0, T ;\left[H^{1}\left(\Omega^{M}\right)\right]^{3}\right), & w_{t} \in L^{\infty}\left(0, T ;\left[L^{2}\left(\Omega^{M}\right)\right]^{3}\right), \\
u \in L^{\infty}\left(0, T ;\left[H^{1}\left(\Omega^{P}\right)\right]^{3}\right), & u_{t} \in L^{\infty}\left(0, T ;\left[L^{2}\left(\Omega^{P}\right)\right]^{3}\right), \\
q \in L^{\infty}\left(0, T ; H^{1}\left(\Omega^{P}\right)\right) . &
\end{array}
$$


In addition, if we assume that $q^{P}(t) \in C^{2}\left(\Gamma_{1}\right)$ and the initial data satisfy the compatibility conditions $\left(\varphi_{0}, w_{0}, u_{0}, \varphi_{1}, w_{1}, u_{1}\right) \in \widehat{\mathcal{W}}$, with

$$
\begin{aligned}
& \widehat{\mathcal{W}}=\left\{\left(\varphi_{0}, w_{0}, u_{0}\right) \in H^{2}\left(\Omega^{A}\right) \times\left[H^{2}\left(\Omega^{M}\right)\right]^{3} \times\left[H^{2}\left(\Omega^{P}\right)\right]^{3},\right. \\
& \left(\varphi_{1}, w_{1}, u_{1}, 0\right) \in \widetilde{W}(\Omega): \\
& \widehat{u}=0, \psi_{0} \cdot n=0 \text { on } \Gamma_{0}, \widehat{w}=\widehat{u}, \sigma_{0} n=S_{0} n \text { on } \Gamma_{1} \text { and } \widehat{q}=0 \text { on } \Gamma_{1} \\
& \left.w_{1} \cdot n=-\frac{\partial}{\partial_{n}} \varphi_{0}, S_{0} n=-\varphi_{1} n \text { on } \Gamma_{2}, \frac{\partial}{\partial n} \varphi_{0}=-\frac{1}{c} \varphi_{1} \text { on } \Gamma_{3}\right\},
\end{aligned}
$$

then the solution belongs to the (more regular) class

$$
\begin{aligned}
& \varphi \in L^{\infty}\left(0, T ; H^{2}\left(\Omega^{A}\right)\right), \quad \varphi_{t} \in L^{\infty}\left(0, T ; H^{1}\left(\Omega^{A}\right)\right), \\
& \varphi_{t t} \in L^{\infty}\left(0, T ; L^{2}\left(\Omega^{A}\right)\right), \\
& w \in L^{\infty}\left(0, T ;\left[H^{2}\left(\Omega^{M}\right)\right]^{3}\right), \quad w_{t} \in L^{\infty}\left(0, T ;\left[H^{1}\left(\Omega^{M}\right)\right]^{3}\right), \\
& w_{t t} \in L^{\infty}\left(0, T ;\left[L^{2}\left(\Omega^{M}\right)\right]^{3}\right), \\
& u \in L^{\infty}\left(0, T ;\left[H^{2}\left(\Omega^{P}\right)\right]^{3}\right), \\
& u_{t t} \in L^{\infty}\left(0, T ;\left[L^{2}\left(\Omega^{P}\right)\right]^{3}\right), \\
& q \in L^{\infty}\left(0, T ; H^{2}\left(\Omega^{P}\right)\right) .
\end{aligned}
$$

Proof The proof of Theorem 1 is relegated to the Appendix.

Remark 2 We can replace $\widetilde{\varphi}$ with $c^{2} \widetilde{\varphi}$ in (3.4) which amounts to multiplying the first equation in (2.1) by $c^{2}$. This is the form used in the sequel.

\subsection{Outlines of the Shape Sensitivity Analysis}

Theorem 1 implies the existence and the regularity of solutions to the model as well as to the systems which are obtained for material and shape derivatives as well as for the adjoint state.

- If the solution of state equation belongs to the class (3.10), then all boundary conditions for the shape derivatives are well defined.

- For the shape functional under consideration the shape differentiability is achieved for the material derivatives belonging to the class (3.8).

- Once the existence of the material derivatives is established for the model, the existence of shape derivatives follows from the relation (4.10).

- If material derivatives belong to the class (3.10), then the shape derivatives belong to the class (3.8).

- If material derivatives belong to the class (3.8) then, in view of (4.10), the shape derivatives are given by very weak solution of the system. 
Therefore, what we really need for the proof of shape differentiability of the functional, is the existence of the regular solution to the model, and the existence of sufficiently smooth material derivatives which can be used in order to obtain the shape differentiability of the functional. The adjoint state allows us to simplify the form of the shape gradient, but there is no implication of the adjoint state on the shape differentiability of the functional. The existence of material derivatives implies the existence of the shape derivatives as well as the differentiability of the shape functional by means of the Hadamard structure Theorem [12] for the shape gradient.

\section{Shape Sensitivity Analysis}

Formal derivation of the coupled equations for shape derivatives of solutions to the model under considerations leads to the shape gradient of the cost functional. In this derivation the transmission conditions on the interfaces should be taken into account, it means that the derivatives with respect to the shape parameter $\tau \rightarrow 0$ are evaluated from both sides of the interface. In our model one exterior boundary $\Gamma_{0}$ and one interface $\Gamma_{1}$ move according to the boundary perturbations rule defined by the speed velocity method. In formal derivation no attention is payed to the regularity of solutions, however we are interested in the resulting shape gradient regularity since the regularity has the important implications on the numerical methods. If the shape gradient is given by a distribution which lives on the moving boundaries or interfaces, this property should be taken into account when computing numerically the descent direction for gradient type numerical methods of shape optimization. On the other hand the levelset methods for shape optimization require the shape gradient of the cost given by a function, the shape gradient becomes the coefficient of the associated Hamilton-Jacoby equations for the levelset function.

On the other hand, the proof of the shape differentiability of the cost functional relies on the material derivatives of solutions to the model. The stability analysis of the model which results in the material derivatives is performed in the fixed domain setting. In this way the shape gradient of the continuous shape functional is precisely determined and it can be used for numerical computations.

For sake of simplicity, in this section we consider that the $f, g$ and $h$ in (2.1) are identically zero. We also consider that the initial conditions (2.4) are homogeneous. We observe that the only source in the system is given by $q=q^{P}(x, t)$ on $\Gamma_{1} \times(0, T)$, which satisfies the compatibility condition, namely, $q^{P}(x, 0)=q_{t}^{P}(x, 0)=0$.

The perturbed domain, parameterized by $\tau \in \mathbb{R}^{+}$small enough, is denoted as

$$
\Omega_{\tau}=\left\{x_{\tau} \in \mathbb{R}^{3}: x_{\tau}=x+\tau V, x \in \Omega, \tau \geq 0\right\},
$$

where $V$ is a smooth vector field defined in $\Omega$ that represents the shape change velocity. Thus, the original domain is retrieved by setting $\tau=0$, that is $\Omega_{0} \equiv \Omega$. In particular, we are interested in the perturbations of the boundary $\Gamma_{0}$ of the electromechanical device and of the interface $\Gamma_{1}$ between the mechanical and electromechanical devices. It means that the shape change velocity field can be defined as

$$
V=0 \quad \text { on } \Gamma_{2} \cup \Gamma_{3}=\partial \Omega^{A} .
$$


The shape functional defined in the perturbed domain reads

$$
\mathcal{J}_{\Omega_{\tau}}\left(\varphi_{\tau, t}, w_{\tau}\right)=\int_{0}^{T} J_{\Omega_{\tau}}\left(\varphi_{\tau, t}, w_{\tau}\right),
$$

where $\varphi_{\tau}=\varphi_{\tau}\left(x_{\tau}, t\right)$ and $w_{\tau}=w_{\tau}\left(x_{\tau}, t\right)$, together with $u_{\tau}=u_{\tau}\left(x_{\tau}, t\right)$ and $q_{\tau}=$ $q_{\tau}\left(x_{\tau}, t\right)$, are solutions of the following variational problem defined in the perturbed domain $\Omega_{\tau}$ : for each $t \in(0, T)$ and any $(\widetilde{\varphi}, \widetilde{w}, \widetilde{u}, \widetilde{q}) \in \widetilde{\mathcal{W}}\left(\Omega_{\tau}\right)$, find

$$
\left(\varphi_{\tau}, w_{\tau}, u_{\tau}, q_{\tau}\right) \in \mathcal{W}\left(\Omega_{\tau}\right)
$$

such that

$$
\left\{\begin{array}{l}
\left\langle\varphi_{\tau, t t}, \widetilde{\varphi}\right\rangle_{\Omega^{A}}+c^{2}\left\langle\nabla \varphi_{\tau}, \nabla \widetilde{\varphi}\right\rangle_{\Omega^{A}}-c^{2}\left\langle w_{\tau, t} \cdot n, \widetilde{\varphi}\right\rangle_{\Gamma_{2}}+c\left\langle\varphi_{\tau, t}, \widetilde{\varphi}\right\rangle_{\Gamma_{3}} \\
\quad+\left\langle w_{\tau, t t}, \widetilde{w}\right\rangle_{\Omega^{M}}+\left\langle A \nabla_{\tau}^{s} w_{\tau}, \nabla_{\tau}^{s} \widetilde{w}\right\rangle_{\Omega^{M}}+\left\langle\varphi_{\tau, t}, \widetilde{w} \cdot n\right\rangle_{\Gamma_{2}} \\
\quad+\left\langle u_{\tau, t t}, \widetilde{u}\right\rangle_{\Omega^{P}}+\left\langle C \nabla_{\tau}^{s} u_{\tau}, \nabla_{\tau}^{s} \widetilde{u}\right\rangle_{\Omega^{P}}+\left\langle P \nabla_{\tau} q_{\tau}, \nabla_{\tau}^{s} \widetilde{u}\right\rangle_{\Omega^{P}}=0 \\
\left\langle D \nabla_{\tau} q_{\tau}, \nabla_{\tau} \widetilde{q}\right\rangle_{\Omega^{P}}-\left\langle P^{\top} \nabla_{\tau}^{s} u_{\tau}, \nabla_{\tau} \widetilde{q}\right\rangle_{\Omega^{P}}=0
\end{array}\right.
$$

with homogeneous initial conditions. In addition, the sets $\mathcal{W}\left(\Omega_{\tau}\right)$ and $\tilde{\mathcal{W}}\left(\Omega_{\tau}\right)$ are defined analogously as before.

\subsection{Material Derivatives of Solutions}

We are going to evaluate material and shape derivatives for the state system, and two formulae for the shape gradient including the distributed representation and the boundary representation. Before start, let us introduce the following notation for material derivative of a function $\xi(x)$

$$
\dot{\xi}(x)=\left.\frac{d}{d \tau} \xi_{\tau}\left(x_{\tau}\right)\right|_{\tau=0} .
$$

We assume for the sake of simplicity that the only source in the system is given by $q=q^{P}(x, t)$ on $\Gamma_{1} \times(0, T)$, which satisfies the compatibility condition $q^{P}(x, 0)=$ $q_{t}^{P}(x, 0)=0$. In addition, we have the nonhomogeneous initial conditions for all functions.

For each $t \in(0, T)$ and any $(\widetilde{\varphi}, \widetilde{w}, \widetilde{u}, \widetilde{q}) \in \tilde{\mathcal{W}}$, find the acoustic potential $\varphi$, the mechanical displacement $w$, the electromechanical displacement $u$ and the electric potential $q$, with $(\varphi, w, u, q) \in \mathcal{W}$, such that

$$
\left\{\begin{array}{l}
\left\langle\varphi_{t t}, \widetilde{\varphi}\right\rangle_{\Omega^{A}}+c^{2}\langle\nabla \varphi, \nabla \widetilde{\varphi}\rangle_{\Omega^{A}}-c^{2}\left\langle w_{t} \cdot n, \widetilde{\varphi}\right\rangle_{\Gamma_{2}}+c\left\langle\varphi_{t}, \widetilde{\varphi}\right\rangle_{\Gamma_{3}} \\
\quad+\left\langle w_{t t}, \widetilde{w}\right\rangle_{\Omega^{M}}+\left\langle A \nabla^{s} w, \nabla^{s} \widetilde{w}\right\rangle_{\Omega^{M}}+\left\langle\varphi_{t}, \widetilde{w} \cdot n\right\rangle_{\Gamma_{2}} \\
\quad+\left\langle u_{t t}, \widetilde{u}\right\rangle_{\Omega^{P}}+\left\langle C \nabla^{s} u, \nabla^{s} \widetilde{u}\right\rangle_{\Omega^{P}}+\left\langle P \nabla q, \nabla^{s} \widetilde{u}\right\rangle_{\Omega^{P}}=0, \\
\langle D \nabla q, \nabla \widetilde{q}\rangle_{\Omega^{P}}-\left\langle P^{\top} \nabla^{s} u, \nabla \widetilde{q}\right\rangle_{\Omega^{P}}=0 .
\end{array}\right.
$$

Beside the above system, for the state equation the initial and boundary conditions are imposed, and the potential $q^{P}$ is prescribed on $\Gamma_{1} \times(0, T)$. 
The material derivatives are given by the solutions to the following system

$$
\left\{\begin{aligned}
\left\langle\dot{\varphi}_{t t},\right. & \widetilde{\varphi}\rangle_{\Omega^{A}}+c^{2}\langle\nabla \dot{\varphi}, \nabla \widetilde{\varphi}\rangle_{\Omega^{A}}-c^{2}\left\langle\dot{w}_{t} \cdot n, \widetilde{\varphi}\right\rangle_{\Gamma_{2}}+c\left\langle\dot{\varphi}_{t}, \widetilde{\varphi}\right\rangle_{\Gamma_{3}} \\
& +\left\langle\dot{w}_{t t}, \widetilde{w}\right\rangle_{\Omega^{M}}+\left\langle A \nabla^{s} \dot{w}, \nabla^{s} \widetilde{w}\right\rangle_{\Omega^{M}}+\langle\dot{\varphi} t, \widetilde{w} \cdot n\rangle_{\Gamma_{2}} \\
& +\left\langle\dot{u}_{t t}, \widetilde{u}\right\rangle_{\Omega^{P}}+\left\langle C \nabla^{s} \dot{u}, \nabla^{s} \widetilde{u}\right\rangle_{\Omega^{P}}+\left\langle P \nabla \dot{q}, \nabla^{s} \widetilde{u}\right\rangle_{\Omega^{P}} \\
= & \left\langle\nabla w^{\top}\left(A \nabla^{s} \widetilde{w}\right)+\nabla \widetilde{w}^{\top}\left(A \nabla^{s} w\right), \nabla V\right\rangle_{\Omega^{M}} \\
& -\left\langle w_{t t} \cdot \widetilde{w}+A \nabla^{s} w \cdot \nabla^{s} \widetilde{w}, \operatorname{div} V\right\rangle_{\Omega^{M}} \\
& +\left\langle\nabla u^{\top}\left(C \nabla^{s} \widetilde{u}\right)+\nabla \widetilde{u}^{\top}\left(A \nabla^{s} u\right)+\nabla q \otimes P^{\top} \nabla^{s} \widetilde{u}+\nabla \widetilde{u}^{\top} P \nabla q, \nabla V\right\rangle_{\Omega^{P}} \\
& -\left\langle u_{t t} \cdot \widetilde{u}+C \nabla^{s} u \cdot \nabla^{s} \widetilde{u}+P \nabla q \cdot \nabla^{s} \widetilde{u}, \operatorname{div} V\right\rangle_{\Omega^{P}}, \\
\langle D \nabla & \dot{q}, \nabla \widetilde{q}\rangle_{\Omega^{P}}-\left\langle P^{\top} \nabla^{s} \dot{u}, \nabla \widetilde{q}\right\rangle_{\Omega^{P}} \\
= & \left\langle\nabla q \otimes D \nabla \widetilde{q}+\nabla \widetilde{q} \otimes D \nabla q-\nabla u^{\top} P \nabla \widetilde{q}-\nabla \widetilde{q} \otimes P^{\top} \nabla^{s} u, \nabla V\right\rangle_{\Omega^{P}} \\
& -\left\langle D \nabla q \cdot \nabla \widetilde{q}-P^{\top} \nabla^{s} u \cdot \nabla \widetilde{q}, \operatorname{div} V\right\rangle_{\Omega^{P}} .
\end{aligned}\right.
$$

The system becomes closed provided we complement the system (4.7) with the following initial conditions

$$
\begin{cases}\dot{\varphi}(x, 0)=\nabla \varphi_{0}(x) \cdot V(x, 0), & \dot{\varphi}_{t}(x, 0)=\nabla \varphi_{1}(x) \cdot V(x, 0), \\ \dot{w}(x, 0)=\nabla w_{0}(x) V(x, 0), & \dot{w}_{t}(x, 0)=\nabla w_{1}(x) V(x, 0), \\ \dot{u}(x, 0)=\nabla u_{0}(x) V(x, 0), & \dot{u}_{t}(x, 0)=\nabla u_{1}(x) V(x, 0),\end{cases}
$$

and boundary conditions

$$
\left\{\begin{array}{l}
\dot{\psi} \cdot n=-\psi \cdot \dot{n} \\
\dot{u}=0
\end{array} \quad \text { on } \Gamma_{0} \times(0, T), \quad \frac{\partial \dot{\varphi}}{\partial n}=-\frac{1}{c} \dot{\varphi}_{t} \quad \text { on } \Gamma_{3} \times(0, T) .\right.
$$

In addition, the potential $\nabla q^{P}(x, t) \cdot V(x, 0)$ is prescribed on $\Gamma_{1} \times(0, T)$ for the material derivative of the electric potential $\dot{q}$.

Theorem 3 The material derivatives of solutions for the system (4.6) are given by (4.7) along with the initial conditions (4.8) and boundary conditions (4.9).

Proof The proof of Theorem 3 is given in Sect. 5.3.

\subsection{Shape Derivatives of Solutions}

The system of equations with the initial and boundary conditions is derived for the shape derivatives of solutions to the model. The shape derivatives lead to the shape gradient of the cost functional. By the Hadamard representation Theorem of the shape gradient, it follows that it is a distribution which lives on the moving boundary. From the point of view of numerical methods of shape optimization, it is preferable to have the shape gradient given by a function. The shape derivatives are given by solutions to the linearized equations with respect to the shape by using the speed method. The initial and boundary value problem for the linearized equations, in view of the 
shape functional under considerations, lead to appropriate adjoint state equations. All together the obtained system defines the regularity of the shape gradient which is expressed in terms of the shape derivatives, the adjoint state and the integrand of the shape functional. By the regularity assumptions on the data, the sufficient regularity of the shape gradient can be achieved. In fact, the regularity of the data is also required for derivation of the shape gradient using the material derivatives. Roughly speaking, the proof of shape differentiability is performed in the material derivatives framework in the fixed domain setting. However, in general, the shape gradient identification is possible with the shape derivatives.

Condition 4 In this section the normal component

$$
v_{n}:=V \cdot n
$$

of the velocity vector field is nonnull only on the boundary $\Gamma_{0}$ and on the interface $\Gamma_{1}$. It means that only $\Gamma_{0}$ and $\Gamma_{1}$ are perturbed by an action of the shape velocity field $V$.

We have the following relation between material and shape derivatives, since in general case the material derivative of a function $\xi$ can be written as

$$
\dot{\xi}=\xi^{\prime}+\langle\nabla \xi, V\rangle
$$

From relation (4.10) it follows that the shape derivatives looses the spatial regularity compared to the material derivatives. For hyperbolic problems this property should be taken into account in order to assure the regularity of shape derivatives in terms of the regularity of the data to the state equation.

The shape derivatives satisfy the homogeneous system (2.1) of the form

$$
\begin{cases}\varphi_{t t}^{\prime}-c^{2} \Delta \varphi^{\prime}=0 & \text { in } \Omega^{A} \times(0, T), \\ w_{t t}^{\prime}-\operatorname{div} S^{\prime}=0 & \text { in } \Omega^{M} \times(0, T), \\ u_{t t}^{\prime}-\operatorname{div} \sigma^{\prime}=0 & \\ -\operatorname{div} \psi^{\prime}=0 & \text { in } \Omega^{P} \times(0, T),\end{cases}
$$

along with the homogeneous initial conditions,

$$
\left\{\begin{array}{l}
\varphi^{\prime}(x, 0)=0, \quad \varphi_{t}^{\prime}(x, 0)=0, \\
w^{\prime}(x, 0)=0, \quad w_{t}^{\prime}(x, 0)=0 \\
u^{\prime}(x, 0)=0, \quad u_{t}^{\prime}(x, 0)=0
\end{array}\right.
$$

and nonhomogeneous boundary and interface conditions obtained below from (2.5) on $\Gamma_{0}$ and from (2.6) on $\Gamma_{1}$, respectively.

The constitutive relations (2.2) are in the same form for the shape derivatives, therefore are not repeated here. 
Boundary Conditions for Shape Derivatives on $\Gamma_{0} \quad$ Now, we derive the boundary conditions on $\Gamma_{0}$.

- The homogeneous Dirichlet boundary condition for the displacement field $u=0$ leads to the homogeneous boundary condition for the material derivative, and in view of (4.10) becomes the nonhomogeneous boundary condition for the shape derivative

$$
u^{\prime}=-\frac{\partial u}{\partial n} V \cdot n=-v_{n} \frac{\partial u}{\partial n} \quad \text { on } \Gamma_{0} \times(0, T) .
$$

- The homogeneous Dirichlet boundary condition for the normal component of the vector field $\psi$ written in the form $\psi_{\tau}\left(x_{\tau}\right) \cdot n_{\tau}\left(x_{\tau}\right)=0$ becomes the nonhomogeneous boundary condition for the normal component of the shape derivative vector field after differentiation with respect to $\tau$,

$$
\psi^{\prime} \cdot n+v_{n} n \cdot D \psi \cdot n-\psi_{\Gamma} \cdot \nabla_{\Gamma} v_{n}=0 \quad \text { on } \Gamma_{0} \times(0, T)
$$

where we denote by $\psi_{\Gamma}:=\psi-(\psi \cdot n) n$ the tangential component of the field $\psi$ on the moving boundary $\Gamma_{0} \times(0, T)$.

- The third condition in (2.5) is just repeated for $\varphi^{\prime}$ since the boundary $\Gamma_{3} \times(0, T)$ is independent of the shape parameter $\tau$.

Boundary Conditions for Shape Derivatives on $\Gamma_{1} \quad$ Now, we derive the transmission conditions on the interface $\Gamma_{1}$.

- The transmission condition for displacement fields $u=w$ leads to nonhomogeneous transmission condition for the shape derivatives obtained in the same way as for homogeneous Dirichlet boundary condition, actually

$$
u^{\prime}+v_{n} \frac{\partial u}{\partial n}=w^{\prime}+v_{n} \frac{\partial w}{\partial n} \quad \text { on } \Gamma_{1} \times(0, T)
$$

- In the similar way the boundary value for the shape derivative $q^{\prime}$ of the potential $q$ is obtained

$$
q^{\prime}+v_{n} \frac{\partial q^{P}}{\partial n}=0 \quad \text { on } \Gamma_{1} \times(0, T) .
$$

- The equality of normal stresses $\sigma n=S n$ on the interface $\Gamma_{1} \times(0, T)$ leads to the nonhomogeneous transmission conditions for normal stresses of shape derivatives $\sigma^{\prime} n, S^{\prime} n$

$$
\begin{aligned}
& \sigma^{\prime} n-v_{n}(h+2 \kappa S n)+\operatorname{div}_{\Gamma}\left(v_{n} \sigma_{\Gamma}\right) \\
& \quad=S^{\prime} n-v_{n}(g+2 \kappa \sigma n)+\operatorname{div}_{\Gamma}\left(v_{n} S_{\Gamma}\right) \text { on } \Gamma_{1} \times(0, T),
\end{aligned}
$$

where $\kappa$ is the mean curvature of $\Gamma_{1}, \sigma_{\Gamma}=\sigma n-(\sigma n \cdot n) n$ is the tangential stress on $\Gamma_{1}, \operatorname{div}_{\Gamma}$ is the tangential divergence on $\Gamma_{1}$, and $S_{\Gamma}=S n-(S n \cdot n) n$ is the tangential stress on $\Gamma_{1}$. 
Therefore, we complement the system (4.11) with the following boundary and transmission conditions

$$
\begin{aligned}
& \left\{\begin{array}{l}
\psi^{\prime} \cdot n=-v_{n} n \cdot D \psi \cdot n+\psi_{\Gamma} \cdot \nabla_{\Gamma} v_{n} \\
u^{\prime}=-v_{n} \frac{\partial u}{\partial n}
\end{array} \quad \text { on } \Gamma_{0} \times(0, T),\right. \\
& \left\{\begin{array}{l}
u^{\prime}+v_{n} \frac{\partial u}{\partial n}=w^{\prime}+v_{n} \frac{\partial w}{\partial n} \\
\sigma^{\prime} n-v_{n}(h+2 \kappa S n)+\operatorname{div}_{\Gamma}\left(v_{n} \sigma_{\Gamma}\right) \\
\quad=S^{\prime} n-v_{n}(g+2 \kappa \sigma n)+\operatorname{div}_{\Gamma}\left(v_{n} S_{\Gamma}\right) \\
q^{\prime}=-v_{n} \frac{\partial q^{P}}{\partial n}
\end{array} \quad \text { on } \Gamma_{1} \times(0, T),\right. \\
& \left\{\begin{array}{l}
w_{t}^{\prime} \cdot n=-\frac{\partial \varphi^{\prime}}{\partial n} \quad \text { on } \Gamma_{2} \times(0, T), \\
S^{\prime} n=-\varphi_{t}^{\prime} n
\end{array}\right. \\
& \frac{\partial \varphi^{\prime}}{\partial n}=-\frac{1}{c} \varphi_{t}^{\prime} \quad \text { on } \Gamma_{3} \times(0, T),
\end{aligned}
$$

where $n$ is the outward unit normal vector pointing toward the exterior of $\Omega$.

Theorem 5 For the shape derivatives of the solutions to the coupled model described in Sect. 2.1, we have:

- The shape derivatives $\varphi^{\prime}, w^{\prime}, u^{\prime}, q^{\prime}$, of the solutions $\varphi, w, u, q$, for the system (4.6) are given by (4.11), (4.12), (4.18)-(4.21) in the strong formulation.

- For the regularity of the weak solutions to this system it is required that the following assumption $q^{D} \in L^{\infty}\left(0, T ; H^{2}\left(\Omega^{P}\right)\right)$ is satisfied, which implies the regularity of the nonhomogeneous Dirichlet boundary condition for the shape derivative $q^{\prime}$,

$$
\frac{\partial q^{D}}{\partial n} V \cdot n \in L^{\infty}\left(0, T ; H^{1 / 2}\left(\Gamma_{1}\right)\right) .
$$

- According to (3.10), (4.10) and (4.22), there exist shape derivatives of the solutions to the system (3.4) with the following regularity

$$
\begin{array}{ll}
\varphi^{\prime} \in L^{\infty}\left(0, T ; H^{1}\left(\Omega^{A}\right)\right), & \varphi_{t}^{\prime} \in L^{\infty}\left(0, T ; L^{2}\left(\Omega^{A}\right)\right), \\
w^{\prime} \in L^{\infty}\left(0, T ;\left[H^{1}\left(\Omega^{M}\right)\right]^{3}\right), & w_{t}^{\prime} \in L^{\infty}\left(0, T ;\left[L^{2}\left(\Omega^{M}\right)\right]^{3}\right), \\
u^{\prime} \in L^{\infty}\left(0, T ;\left[H^{1}\left(\Omega^{P}\right)\right]^{3}\right), & u_{t}^{\prime} \in L^{\infty}\left(0, T ;\left[L^{2}\left(\Omega^{P}\right)\right]^{3}\right), \\
q^{\prime} \in L^{\infty}\left(0, T ; H^{1}\left(\Omega^{P}\right)\right), &
\end{array}
$$

given by weak solutions to the following system:

- equations are given by (4.11);

- initial conditions are homogeneous (4.12);

- boundary and transmission conditions are given by (4.18)-(4.21). 
Proof The proof is standard, taking into account the specificity of the hyperbolic systems, the simplest case of the wave equation is covered in details e.g., by Cagnol and Zolésio [1], see also Sokolowski and Zolésio [12] as well as Delfour and Zolésio [3]. Formally, the equations for the shape derivatives are derived by an application of the Reynolds' Transport Theorem to the variational formulation of the model in variable domain setting. Then, the boundary conditions on moving boundary and moving interface are found from the results given in [12] for the shape derivatives of the elasticity boundary value problems. The initial conditions are derived from the assumption that the initial conditions for the model are shape independent i.e., the shape derivatives of initial conditions are null.

\section{Shape Differentiability of a Functional}

\subsection{Adjoint System}

In order to simplify further calculations, let us introduce the adjoint states $\varphi^{a}, w^{a}, v$ and $p$, which are solutions of the following variational system: For each $t \in(0, T)$ and any $(\widetilde{\varphi}, \widetilde{w}, \widetilde{v}, \widetilde{p}) \in \widetilde{\mathcal{W}}(\Omega)$, find the adjoint acoustic potential $\varphi^{a}$, the adjoint mechanical displacement $w^{a}$, the adjoint electromechanical displacement $v$ and the adjoint electric potential $p$, with $\left(\varphi^{a}, w^{a}, v, p\right) \in \widetilde{\mathcal{W}}$, such that

$$
\left\{\begin{array}{c}
\left\langle\varphi_{t t}^{a}, \widetilde{\varphi}\right\rangle_{\Omega^{A}}+c^{2}\left\langle\nabla \varphi^{a}, \nabla \widetilde{\varphi}\right\rangle_{\Omega^{A}}-\left\langle w_{t}^{a} \cdot n, \widetilde{\varphi}\right\rangle_{\Gamma_{2}}-c\left\langle\varphi_{t}^{a}, \widetilde{\varphi}\right\rangle_{\Gamma_{3}} \\
+\left\langle w_{t t}^{a}, \widetilde{w}\right\rangle_{\Omega^{M}}+\left\langle A \nabla^{s} w^{a}, \nabla^{s} \widetilde{w}\right\rangle_{\Omega^{M}}+c^{2}\left\langle\varphi_{t}^{a}, \widetilde{w} \cdot n\right\rangle_{\Gamma_{2}} \\
\quad+\left\langle v_{t t}, \widetilde{v}\right\rangle_{\Omega^{P}}+\left\langle C \nabla^{s} v, \nabla^{s} \widetilde{v}\right\rangle_{\Omega^{P}}-\left\langle P \nabla p, \nabla^{s} \widetilde{v}\right\rangle_{\Omega^{P}} \\
=\alpha\left\langle\varphi_{t t}-p_{t}^{\star}, \widetilde{\varphi}\right\rangle_{\Omega^{A}}+\beta\left(\langle\eta, \operatorname{div}(\widetilde{w})\rangle_{\Omega^{M}}+\langle\nabla \eta, \widetilde{w}\rangle_{\Omega^{M}},\right. \\
\langle D \nabla p, \nabla \widetilde{p}\rangle_{\Omega^{P}}+\left\langle P^{\top} \nabla^{s} v, \nabla \widetilde{p}\right\rangle_{\Omega^{P}}=0,
\end{array}\right.
$$

with the following final conditions

$$
\begin{aligned}
& \varphi^{a}(x, T)=0 \quad \text { and } \quad \varphi_{t}^{a}(x, T)=-\alpha\left(\varphi_{t}(x, T)-p^{\star}(x, T)\right), \\
& w^{a}(x, T)=w_{t}^{a}(x, T)=0, \quad v(x, T)=v_{t}(x, T)=0 .
\end{aligned}
$$

From the above system, we can define the adjoint mechanical stress tensor $S^{a}$, the electromechanical stress tensor $\sigma^{a}$ and the adjoint electrical displacement $\psi^{a}$ as following

$$
\left\{\begin{array}{l}
S^{a}\left(w^{a}\right)=A \varepsilon\left(w^{a}\right), \\
\sigma^{a}(v, p)=C \varepsilon(v)+P e(p), \\
\psi^{a}(v, p)=-P^{\top} \varepsilon(v)+\operatorname{De}(p) .
\end{array}\right.
$$

The strong system associated to the adjoint problem reads as follows

$$
\begin{cases}\varphi_{t t}^{a}-c^{2} \Delta \varphi^{a}=\alpha\left(\varphi_{t t}-p_{t}^{\star}\right) & \text { in } \Omega^{A} \times(0, T), \\ w_{t t}^{a}-\operatorname{div} S^{a}=0 & \text { in } \Omega^{M} \times(0, T), \\ v_{t t}-\operatorname{div} \sigma^{a}=0 & \text { in } \Omega^{P} \times(0, T), \\ -\operatorname{div} \psi^{a}=0 & \end{cases}
$$


with final conditions given by (5.2), boundary conditions

$$
\left\{\begin{array}{l}
\psi^{a} \cdot n=0 \\
v=0
\end{array} \quad \text { on } \Gamma_{0} \times(0, T), \quad \frac{\partial \varphi^{a}}{\partial n}=\frac{1}{c} \varphi_{t}^{a} \quad \text { on } \Gamma_{3} \times(0, T),\right.
$$

and transmission conditions of the form

$$
\begin{aligned}
& \left\{\begin{array}{l}
v=w^{a} \\
\left(\sigma^{a}-S^{a}\right) n=-\beta \eta n
\end{array} \quad \text { on } \Gamma_{1} \times(0, T) \quad\right. \text { and } \\
& \left\{\begin{array}{l}
w_{t}^{a} \cdot n=-c^{2} \frac{\partial \varphi^{a}}{\partial n} \\
S^{a} n=\left(\beta \eta-c^{2} \varphi_{t}^{a}\right) n
\end{array} \quad \text { on } \Gamma_{2} \times(0, T) .\right.
\end{aligned}
$$

In addition, we have $p(x, t)=0$ on $\Gamma_{1} \times(0, T)$, which naturally satisfies the compatibility condition.

Remark 6 It is important to observe that the adjoint system is a time reversal problem, which should be solved by taking $t \leftarrow T-t$. In this case the boundary condition on $\Gamma_{3}$ becomes dissipative, namely

$$
\frac{\partial \varphi^{a}}{\partial n}=-\frac{1}{c} \varphi_{t}^{a} \quad \text { on } \Gamma_{3} \times(0, T),
$$

and for the adjoint system holds Theorem 1 .

Proposition 1 There is a unique weak solution $\left(\varphi^{a}, w^{a}, v, p\right)$ satisfying the regularity (3.8) of Theorem 1 for the adjoint system (5.4), (5.2), (5.5), (5.6).

\subsection{Shape Derivative Calculation}

We are going to denote by $\varphi_{\tau, t}:=\frac{\partial \varphi_{\tau}}{\partial t}$ the time derivative of the function $\varphi_{\tau}$ which is defined in $\Omega_{\tau}$.

Let us perform the shape sensitivity analysis of the functional $\mathcal{J}_{\Omega_{\tau}}\left(\varphi_{\tau, t}, w_{\tau}\right)$. Thus, we need to calculate its derivative with respect to the parameter $\tau$ at $\tau=0$, that is

$$
\int_{0}^{T} \dot{J}_{\Omega}\left(\varphi_{t}, w\right)=\dot{\mathcal{J}}_{\Omega}\left(\varphi_{t}, w\right):=\left.\frac{d}{d \tau} \mathcal{J}_{\Omega_{\tau}}\left(\varphi_{\tau, t}, w_{\tau}\right)\right|_{\tau=0} .
$$

In order to proceed, it is convenient to introduce an analogy to classical continuum mechanics [6] whereby the shape change velocity field $V$ is identified with the classical velocity field of a deforming continuum and $\tau$ is identified as an artificial time parameter (we refer to [12] for analogies of this type in the context of shape sensitivity analysis). In this case, by making use of the concept of material derivative of a spatial field $[6,7]$ and considering the Reynolds' Transport Theorem, the shape 
derivative of the functional $J_{\Omega}\left(\varphi_{t}, w\right)$ is given by

$$
\dot{J}_{\Omega}\left(\varphi_{t}, w\right)=\left\langle D_{\Omega}\left(J_{\Omega}\left(\varphi_{t}, w\right)\right), V\right\rangle+\left\langle D_{\varphi_{t}}\left(J_{\Omega}\left(\varphi_{t}, w\right)\right), \dot{\varphi}_{t}\right\rangle+\left\langle D_{w}\left(J_{\Omega}\left(\varphi_{t}, w\right)\right), \dot{w}\right\rangle,
$$

where

$$
\begin{aligned}
\int_{0}^{T}\left\langle D_{\Omega}\left(J_{\Omega}\left(\varphi_{t}, w\right)\right), V\right\rangle= & \beta \int_{0}^{T} \int_{\Omega^{M}}\left(\nabla w^{\top} \eta+\nabla \eta \otimes w\right) \cdot \nabla V \\
& -\beta \int_{0}^{T} \int_{\Omega^{M}}(\operatorname{div}(w) \eta+w \cdot \nabla \eta) \operatorname{div} V \\
= & \beta \int_{0}^{T}\left\langle\nabla w^{\top} \eta+\nabla \eta \otimes w, \nabla V\right\rangle_{\Omega^{M}} \\
& -\beta \int_{0}^{T}\langle\operatorname{div}(w) \eta+w \cdot \nabla \eta, \operatorname{div} V\rangle_{\Omega^{M}},
\end{aligned}
$$

and

$$
\begin{aligned}
\int_{0}^{T}\left\langle D_{\varphi_{t}}\left(J_{\Omega}\left(\varphi_{t}, w\right)\right), \dot{\varphi}_{t}\right\rangle & =\alpha \int_{0}^{T} \int_{\Omega^{A}}\left(\varphi_{t}-p^{\star}\right) \dot{\varphi}_{t} \\
& =\left.\alpha \int_{\Omega^{A}}\left(\varphi_{t}-p^{\star}\right) \dot{\varphi}\right|_{0} ^{T}-\alpha \int_{0}^{T} \int_{\Omega^{A}}\left(\varphi_{t t}-p_{t}^{\star}\right) \dot{\varphi} \\
& =\alpha \int_{\Omega^{A}}\left(\varphi_{t}(T)-p^{\star}(T)\right) \dot{\varphi}(T)-\alpha \int_{0}^{T} \int_{\Omega^{A}}\left(\varphi_{t t}-p_{t}^{\star}\right) \dot{\varphi} \\
& =\alpha\left\langle\left(\varphi_{t}(T)-p^{\star}(T)\right), \dot{\varphi}(T)\right\rangle_{\Omega^{A}}-\alpha \int_{0}^{T}\left\langle\left(\varphi_{t t}-p_{t}^{\star}\right), \dot{\varphi}\right\rangle_{\Omega^{A}},
\end{aligned}
$$

$$
\begin{aligned}
\int_{0}^{T}\left\langle D_{w}\left(J_{\Omega}\left(\varphi_{t}, w\right)\right), \dot{w}\right\rangle & =-\beta \int_{0}^{T} \int_{\Omega^{M}}(\operatorname{div}(\dot{w}) \eta+\dot{w} \cdot \nabla \eta) \\
& =-\beta \int_{0}^{T}\left(\langle\eta, \operatorname{div}(\dot{w})\rangle_{\Omega^{M}}+\langle\nabla \eta, \dot{w}\rangle_{\Omega^{M}}\right) .
\end{aligned}
$$

Thus, since the acoustic chamber remains fixed, we have

$$
\begin{aligned}
\dot{\mathcal{J}}_{\Omega}\left(\varphi_{t}, w\right)= & \beta \int_{0}^{T}\left\langle\nabla w^{\top} \eta+\nabla \eta \otimes w, \nabla V\right\rangle_{\Omega^{M}}-\beta \int_{0}^{T}\langle\operatorname{div}(w) \eta+w \cdot \nabla \eta, \operatorname{div} V\rangle_{\Omega^{M}} \\
& -\int_{0}^{T} \alpha\left\langle\left(\varphi_{t t}-p_{t}^{\star}\right), \dot{\varphi}\right\rangle_{\Omega^{A}}-\int_{0}^{T} \beta\left(\langle\eta, \operatorname{div}(\dot{w})\rangle_{\Omega^{M}}+\langle\nabla \eta, \dot{w}\rangle_{\Omega^{M}}\right. \\
& +\alpha\left\langle\left(\varphi_{t}(T)-p^{\star}(T)\right), \dot{\varphi}(T)\right\rangle_{\Omega^{A}}
\end{aligned}
$$




\subsection{Proof of Theorem 3}

Let us now calculate the derivative of the state system (4.4) with respect to the parameter $\tau$ at $\tau=0$. Thus, by making use again of the concept of material derivative of a spatial field $[6,7]$ and considering the Reynolds' Transport theorem, we obtain:

- For the acoustic chamber

$$
\begin{aligned}
\left\langle\varphi_{t t}, \widetilde{\varphi}\right\rangle_{\Omega^{A}} & =\left\langle\dot{\varphi}_{t t}, \widetilde{\varphi}\right\rangle_{\Omega^{A}}, \\
\langle\nabla \varphi, \nabla \widetilde{\varphi}\rangle_{\Omega^{A}} & =\langle\nabla \dot{\varphi}, \nabla \widetilde{\varphi}\rangle_{\Omega^{A}}, \\
\left\langle w_{t} \cdot n, \widetilde{\varphi}\right\rangle_{\Gamma_{2}} & =\left\langle\dot{w}_{t} \cdot n, \widetilde{\varphi}\right\rangle_{\Gamma_{2}}, \\
\left\langle\varphi_{t}, \widetilde{\varphi}\right\rangle_{\Gamma_{3}} & =\left\langle\dot{\varphi}_{t}, \widetilde{\varphi}\right\rangle_{\Gamma_{3}} .
\end{aligned}
$$

- For the mechanical device

$$
\begin{aligned}
\left\langle w_{t t}, \widetilde{w}\right\rangle_{\Omega^{M}} & =\left\langle\dot{w}_{t t}, \widetilde{w}\right\rangle_{\Omega^{M}}+\int_{\Omega^{M}}\left(w_{t t} \cdot \widetilde{w}\right) \operatorname{div} V, \\
\left\langle\varphi_{t}, \widetilde{w} \cdot n\right\rangle_{\Gamma_{2}}= & \left\langle\dot{\varphi}_{t}, \widetilde{w} \cdot n\right\rangle_{\Gamma_{2}}, \\
\left\langle A \nabla^{s} w, \nabla^{s} \widetilde{w}\right\rangle_{\Omega^{M}}= & \left\langle A \nabla^{s} \dot{w}, \nabla^{s} \widetilde{w}\right\rangle_{\Omega^{M}}+\int_{\Omega^{M}}\left(A \nabla^{s} w \cdot \nabla^{s} \widetilde{w}\right) \operatorname{div} V \\
& -\int_{\Omega^{M}}\left(\nabla w^{\top}\left(A \nabla^{s} \widetilde{w}\right)+\nabla \widetilde{w}^{\top}\left(A \nabla^{s} w\right)\right) \cdot \nabla V .
\end{aligned}
$$

- For the piezoelectric device

$$
\begin{aligned}
& \left\langle u_{t t}, \tilde{u}\right\rangle_{\Omega^{P}}=\left\langle\dot{u}_{t t}, \tilde{u}\right\rangle_{\Omega^{P}}+\int_{\Omega^{P}}\left(u_{t t} \cdot \widetilde{u}\right) \operatorname{div} V, \\
& \left\langle C \nabla^{s} u, \nabla^{s} \widetilde{u}\right\rangle_{\Omega^{P}}=\left\langle C \nabla^{s} \dot{u}, \nabla^{s} \widetilde{u}\right\rangle_{\Omega^{P}}+\int_{\Omega^{P}}\left(C \nabla^{s} u \cdot \nabla^{s} \widetilde{u}\right) \operatorname{div} V \\
& -\int_{\Omega^{P}}\left(\nabla u^{\top}\left(C \nabla^{s} \widetilde{u}\right)+\nabla \tilde{u}^{\top}\left(A \nabla^{s} u\right)\right) \cdot \nabla V \\
& \left\langle P \nabla q, \nabla^{s} \widetilde{u}\right\rangle_{\Omega^{P}}=\left\langle P \nabla \dot{q}, \nabla^{s} \widetilde{u}\right\rangle_{\Omega^{P}}+\int_{\Omega^{P}}\left(P \nabla q \cdot \nabla^{s} \widetilde{u}\right) \operatorname{div} V \\
& -\int_{\Omega^{P}}\left(\nabla q \otimes P^{\top} \nabla^{s} \widetilde{u}+\nabla \tilde{u}^{\top} P \nabla q\right) \cdot \nabla V \\
& \langle D \nabla q, \nabla \widetilde{q}\rangle_{\Omega^{P}}=\langle D \nabla \dot{q}, \nabla \widetilde{q}\rangle_{\Omega^{P}}+\int_{\Omega^{P}}(D \nabla q \cdot \nabla \widetilde{q}) \operatorname{div} V \\
& -\int_{\Omega^{P}}(\nabla q \otimes D \nabla \tilde{q}+\nabla \widetilde{q} \otimes D \nabla q) \cdot \nabla V \\
& \left\langle P^{\top} \nabla^{s} u, \nabla \widetilde{q}\right\rangle_{\Omega^{P}}=\left\langle P^{\top} \nabla^{s} \dot{u}, \nabla \widetilde{q}\right\rangle_{\Omega^{P}}+\int_{\Omega^{P}}\left(P^{\top} \nabla^{s} u \cdot \nabla \widetilde{q}\right) \operatorname{div} V \\
& -\int_{\Omega^{P}}\left(\nabla u^{\top} P \nabla \tilde{q}+\nabla \widetilde{q} \otimes P^{\top} \nabla^{s} u\right) \cdot \nabla V
\end{aligned}
$$


where we have used the fact that the admissible variations $\widetilde{\varphi}, \widetilde{w}, \tilde{u}$ and $\widetilde{q}$ do not depend on the parameter $\tau$. Thus, the derivative with respect to the shape parameter $\tau$ of the state system, after some rearrangements, becomes (4.7).

\subsection{Distributed Shape Gradient}

Theorem 7 The form of distributed gradient of shape functional (4.3) defined in variable domain setting, is given by (5.35), (5.36), (5.37). In addition, for the strong solutions we have the divergence free Eshelby tensors (5.46).

Proof By setting in (4.7) $\tilde{\varphi}=\varphi^{a}, \widetilde{w}=w^{a}, \widetilde{u}=v$ and $\widetilde{q}=p$ in the above, we obtain

$$
\left\{\begin{aligned}
\left\langle\varphi_{t t}^{a},\right. & \dot{\varphi}\rangle_{\Omega^{A}}+c^{2}\left\langle\nabla \dot{\varphi}, \nabla \varphi^{a}\right\rangle_{\Omega^{A}}-c^{2}\left\langle\dot{w}_{t} \cdot n, \varphi^{a}\right\rangle_{\Gamma_{2}}+c\left\langle\dot{\varphi}_{t}, \varphi^{a}\right\rangle_{\Gamma_{3}} \\
& +\left\langle w_{t t}^{a}, \dot{w}\right\rangle_{\Omega^{M}}+\left\langle A \nabla^{s} \dot{w}, \nabla^{s} w^{a}\right\rangle_{\Omega^{M}}+\left\langle\dot{\varphi}_{t}, w^{a} \cdot n\right\rangle_{\Gamma_{2}} \\
& +\left\langle v_{t t}, \dot{u}\right\rangle_{\Omega^{P}}+\left\langle C \nabla^{s} \dot{u}, \nabla^{s} v\right\rangle_{\Omega^{P}}+\left\langle P \nabla \dot{q}, \nabla^{s} v\right\rangle_{\Omega^{P}} \\
= & \left\langle\nabla w^{\top}\left(A \nabla^{s} w^{a}\right)+\left(\nabla w^{a}\right)^{\top}\left(A \nabla^{s} w\right), \nabla V\right\rangle_{\Omega^{M}} \\
& -\left\langle w_{t t} \cdot w^{a}+A \nabla^{s} w \cdot \nabla^{s} w^{a}, \operatorname{div} V\right\rangle_{\Omega^{M}} \\
& +\left\langle\nabla u^{\top}\left(C \nabla^{s} v\right)+\nabla v^{\top}\left(A \nabla^{s} u\right)+\nabla q \otimes P^{\top} \nabla^{s} v\right. \\
& \left.+\nabla v^{\top} P \nabla q, \nabla V\right\rangle_{\Omega^{P}} \\
& -\left\langle u_{t t} \cdot v+C \nabla^{s} u \cdot \nabla^{s} v+P \nabla q \cdot \nabla^{s} v, \operatorname{div} V\right\rangle_{\Omega^{P}} \\
& +\left\langle\varphi_{t t}^{a}, \dot{\varphi}\right\rangle_{\Omega^{A}}-\left\langle\dot{\varphi}_{t t}, \varphi^{a}\right\rangle_{\Omega^{A}} \\
& +\left\langle w_{t t}^{a}, \dot{w}\right\rangle_{\Omega^{M}}-\left\langle\dot{w}_{t t}, w^{a}\right\rangle_{\Omega^{M}} \\
& +\left\langle v_{t t}, \dot{u}\right\rangle_{\Omega^{P}}-\left\langle\dot{u}_{t t}, v\right\rangle_{\Omega^{P}}, \\
\langle D \nabla \dot{q} & , \nabla p\rangle_{\Omega^{P}}-\left\langle P^{\top} \nabla^{s} \dot{u}, \nabla p\right\rangle_{\Omega^{P}} \\
= & \left\langle\nabla q \otimes D \nabla p+\nabla p \otimes D \nabla q-\nabla u^{\top} P \nabla p-\nabla p \otimes P^{\top} \nabla^{s} u, \nabla V\right\rangle_{\Omega^{P}} \\
& -\left\langle D \nabla q \cdot \nabla p-P^{\top} \nabla^{s} u \cdot \nabla p, \operatorname{div} V\right\rangle_{\Omega^{P}},
\end{aligned}\right.
$$

where we have introduced the terms $\pm\left\langle\varphi_{t t}^{a}, \dot{\varphi}\right\rangle_{\Omega^{A}}, \pm\left\langle w_{t t}^{a}, \dot{w}\right\rangle_{\Omega^{M}}, \pm\left\langle v_{t t}, \dot{u}\right\rangle_{\Omega^{P}}$ in the left hand side of the first equality. Using integration by parts, we have

$$
\begin{aligned}
\int_{0}^{T}\left\langle\varphi_{t t}^{a}, \dot{\varphi}\right\rangle_{\Omega^{A}}-\int_{0}^{T}\left\langle\dot{\varphi}_{t t}, \varphi^{a}\right\rangle_{\Omega^{A}} & =\left.\left\langle\varphi_{t}^{a}, \dot{\varphi}\right\rangle_{\Omega^{A}}\right|_{0} ^{T}-\left.\left\langle\dot{\varphi}_{t}, \varphi^{a}\right\rangle_{\Omega^{A}}\right|_{0} ^{T} \\
& =\left\langle\varphi_{t}^{a}(T), \dot{\varphi}(T)\right\rangle_{\Omega^{A}} \\
& =-\left\langle\alpha\left(\varphi_{t}(T)-p^{\star}(T)\right), \dot{\varphi}(T)\right\rangle_{\Omega^{A}}, \\
\int_{0}^{T}\left\langle w_{t t}^{a}, \dot{w}\right\rangle_{\Omega^{M}} & =\int_{0}^{T}\left\langle\dot{w}_{t t}, w^{a}\right\rangle_{\Omega^{M}}, \\
\int_{0}^{T}\left\langle v_{t t}, \dot{u}\right\rangle_{\Omega^{P}} & =\int_{0}^{T}\left\langle\dot{u}_{t t}, v\right\rangle_{\Omega^{P}}, \\
\int_{0}^{T}\left\langle\dot{w}_{t} \cdot n, \varphi^{a}\right\rangle_{\Gamma_{2}} & =-\int_{0}^{T}\left\langle\varphi_{t}^{a}, \dot{w} \cdot n\right\rangle_{\Gamma_{2}},
\end{aligned}
$$




$$
\begin{gathered}
\int_{0}^{T}\left\langle\dot{\varphi}_{t}, \varphi^{a}\right\rangle_{\Gamma_{3}}=-\int_{0}^{T}\left\langle\varphi_{t}^{a}, \dot{\varphi}\right\rangle_{\Gamma_{3}}, \\
\int_{0}^{T}\left\langle\dot{\varphi}_{t}, w^{a} \cdot n\right\rangle_{\Gamma_{2}}=-\int_{0}^{T}\left\langle w_{t}^{a} \cdot n, \dot{\varphi}\right\rangle_{\Gamma_{2}} .
\end{gathered}
$$

Thus, identity (5.26) can be re-written as

$$
\left\{\begin{aligned}
\left\langle\varphi_{t t}^{a},\right. & \dot{\varphi}\rangle_{\Omega^{A}}+c^{2}\left\langle\nabla \varphi^{a}, \nabla \dot{\varphi}\right\rangle_{\Omega^{A}}-\left\langle w_{t}^{a} \cdot n, \dot{\varphi}\right\rangle_{\Gamma_{2}}-c\left\langle\varphi_{t}^{a}, \dot{\varphi}\right\rangle_{\Gamma_{3}} \\
& +\left\langle w_{t t}^{a}, \dot{w}\right\rangle_{\Omega^{M}}+\left\langle A \nabla^{s} w^{a}, \nabla^{s} \dot{w}\right\rangle_{\Omega^{M}}+c^{2}\left\langle\varphi_{t}^{a}, \dot{w} \cdot n\right\rangle_{\Gamma_{2}} \\
& +\left\langle v_{t t}, \dot{u}\right\rangle_{\Omega^{P}}+\left\langle C \nabla^{s} v, \nabla^{s} \dot{u}\right\rangle_{\Omega^{P}}-\left\langle P \nabla p, \nabla^{s} \dot{u}\right\rangle_{\Omega^{P}} \\
= & \left\langle\nabla w^{\top}\left(A \nabla^{s} w^{a}\right)+\left(\nabla w^{a}\right)^{\top}\left(A \nabla^{s} w\right), \nabla V\right\rangle_{\Omega^{M}} \\
- & \left\langle w_{t t} \cdot w^{a}+A \nabla^{s} w \cdot \nabla^{s} w^{a}, \operatorname{div} V\right\rangle_{\Omega^{M}} \\
& +\left\langle\nabla u^{\top}\left(C \nabla^{s} v\right)+\nabla v^{\top}\left(A \nabla^{s} u\right)\right. \\
& \left.+\nabla q \otimes P^{\top} \nabla^{s} v+\nabla v^{\top} P \nabla q, \nabla V\right\rangle_{\Omega^{P}} \\
& -\left\langle u_{t t} \cdot v+C \nabla^{s} u \cdot \nabla^{s} v+P \nabla q \cdot \nabla^{s} v, \operatorname{div} V\right\rangle_{\Omega^{P}} \\
& -\left\langle\nabla^{s} v, P \nabla \dot{q}\right\rangle_{\Omega^{P}}-\left\langle P \nabla p, \nabla^{s} \dot{u}\right\rangle_{\Omega^{P}}, \\
\langle D \nabla & p, \nabla \dot{q}\rangle_{\Omega^{P}}+\left\langle P^{\top} \nabla^{s} v, \nabla \dot{q}\right\rangle_{\Omega^{P}} \\
= & \left\langle\nabla q \otimes D \nabla p+\nabla p \otimes D \nabla q-\nabla u^{\top} P \nabla p-\nabla p \otimes P^{\top} \nabla^{s} u, \nabla V\right\rangle_{\Omega^{P}} \\
& -\left\langle D \nabla q \cdot \nabla p-P^{\top} \nabla^{s} u \cdot \nabla p, \operatorname{div} V\right\rangle_{\Omega^{P}} \\
& +\left\langle\nabla p, P^{\top} \nabla^{s} \dot{u}\right\rangle_{\Omega^{P}}+\left\langle P^{\top} \nabla^{s} v, \nabla \dot{q}\right\rangle_{\Omega^{P}},
\end{aligned}\right.
$$

where we have introduced the terms $\pm\left\langle P \nabla p, \nabla^{s} \dot{u}\right\rangle_{\Omega^{P}}$ and $\pm\left\langle P^{\top} \nabla^{s} v, \nabla \dot{q}\right\rangle_{\Omega^{P}}$. In the same way, let us set $\widetilde{\varphi}=\dot{\varphi}, \widetilde{w}=\dot{w}, \widetilde{v}=\dot{u}$ and $\widetilde{p}=\dot{q}$ in the adjoint system (5.1), to obtain

$$
\left\{\begin{array}{c}
\left\langle\varphi_{t t}^{a}, \dot{\varphi}\right\rangle_{\Omega^{A}}+c^{2}\left\langle\nabla \varphi^{a}, \nabla \dot{\varphi}\right\rangle_{\Omega^{A}}-\left\langle w_{t}^{a} \cdot n, \dot{\varphi}\right\rangle_{\Gamma_{2}}-c\left\langle\varphi_{t}^{a}, \dot{\varphi}\right\rangle_{\Gamma_{3}} \\
+\left\langle w_{t t}^{a}, \dot{w}\right\rangle_{\Omega^{M}}+\left\langle A \nabla^{s} w^{a}, \nabla^{s} \dot{w}\right\rangle_{\Omega^{M}}+c^{2}\left\langle\varphi_{t}^{a}, \dot{w} \cdot n\right\rangle_{\Gamma_{2}} \\
+\left\langle v_{t t}, \dot{u}\right\rangle_{\Omega^{P}}+\left\langle C \nabla^{s} v, \nabla^{s} \dot{u}\right\rangle_{\Omega^{P}}-\left\langle P \nabla p, \nabla^{s} \dot{u}\right\rangle_{\Omega^{P}} \\
=\alpha\left\langle\varphi_{t t}-p_{t}^{\star}, \dot{\varphi}\right\rangle_{\Omega^{A}}+\beta\left(\langle\eta, \operatorname{div}(\dot{w})\rangle_{\Omega^{M}}+\langle\nabla \eta, \dot{w}\rangle_{\Omega^{M}},\right. \\
\langle D \nabla p, \nabla \dot{q}\rangle_{\Omega^{P}}+\left\langle P^{\top} \nabla^{s} v, \nabla \dot{q}\right\rangle_{\Omega^{P}}=0 .
\end{array}\right.
$$

By comparing (5.33) with (5.34), and using (5.13) we observe that

$$
\dot{\mathcal{J}}_{\Omega}\left(\varphi_{t}, w\right)=\int_{0}^{\top}\left(\int_{\Omega^{M}} \Sigma^{M} \cdot \nabla V+\int_{\Omega^{P}} \Sigma^{P} \cdot \nabla V\right),
$$

where the last term of (5.13) is absorbed by (5.27) and we have used the fact that $\left\langle\nabla^{s} v, P \nabla \dot{q}\right\rangle_{\Omega^{P}}=\left\langle P^{\top} \nabla^{s} v, \nabla \dot{q}\right\rangle_{\Omega^{P}}$ and $\left\langle P \nabla p, \nabla^{s} \dot{u}\right\rangle_{\Omega^{P}}=\left\langle\nabla p, P^{\top} \nabla^{s} \dot{u}\right\rangle_{\Omega^{P}}$. In ad- 
dition, the Eshelby tensors [4] $\Sigma^{M}$ and $\Sigma^{P}$ are respectively given by

$$
\begin{aligned}
\Sigma^{M}= & -\left(w_{t} \cdot w_{t}^{a}-S \cdot \nabla^{s} w^{a}+\beta(\operatorname{div}(w) \eta+w \cdot \nabla \eta)\right) I \\
& -\left(\nabla w^{\top} S^{a}+\left(\nabla w^{a}\right)^{\top} S-\beta\left(\nabla w^{\top} \eta+\nabla \eta \otimes w\right),\right. \\
\Sigma^{P}= & -\left(u_{t} \cdot v_{t}-\sigma \cdot \nabla^{s} v+\psi \cdot \nabla p\right) I \\
& -\left(\nabla u^{\top} \sigma^{a}+\nabla v^{\top} \sigma-\nabla q \otimes \psi^{a}-\nabla p \otimes \psi\right),
\end{aligned}
$$

with $\sigma, \psi$ and $\sigma^{a}, \psi^{a}$ given, respectively, by (2.2) and (5.3).

\subsection{Boundary Shape Gradient}

Theorem 8 By the structure theorem for a shape differentiable shape functionals [12], from (5.35) the boundary formulae of the shape gradient is obtained. In general, the shape gradient on the boundary is given by a distribution. However, for the strong solutions, in view of (5.46), the boundary formula for the shape gradient takes the form (5.47), and in such a case the shape gradient on the moving boundary is given by a function.

Proof After applying the divergence theorem in (5.35), we observe that

$$
\begin{aligned}
\int_{\Omega^{M}} \Sigma^{M} \cdot \nabla V & =\int_{\partial \Omega^{M}} \Sigma^{M} n \cdot V-\int_{\Omega^{M}} \operatorname{div} \Sigma^{M} \cdot V \\
& =\int_{\Gamma_{2}} \Sigma^{M} n \cdot V-\int_{\Gamma_{1}} \Sigma^{M} n \cdot V-\int_{\Omega^{M}} \operatorname{div} \Sigma^{M} \cdot V, \\
\int_{\Omega^{P}} \Sigma^{P} \cdot \nabla V & =\int_{\partial \Omega^{P}} \Sigma^{P} n \cdot V-\int_{\Omega^{P}} \operatorname{div} \Sigma^{P} \cdot V \\
& =\int_{\Gamma_{1}} \Sigma^{P} n \cdot V-\int_{\Gamma_{0}} \Sigma^{P} n \cdot V-\int_{\Omega^{P}} \operatorname{div} \Sigma^{P} \cdot V,
\end{aligned}
$$

remembering that $n=n^{(i)}=-n^{(i-1)}$ is the unit normal vector pointing toward the exterior of $\mathcal{B}_{i}$. Let us calculate the divergence of the tensors $\Sigma^{M}$ and $\Sigma^{P}$ given by (5.36) and (5.37), respectively

$$
\begin{aligned}
\operatorname{div} \Sigma^{M}= & \nabla w_{t t}^{\top} w^{a}+\left(\nabla w^{a}\right)^{\top} w_{t t}-\nabla w^{\top} \operatorname{div} S^{a}-\left(\nabla w^{a}\right)^{\top} \operatorname{div} S . \\
\operatorname{div} \Sigma^{P}= & \nabla u_{t t}^{\top} v+\nabla v^{\top} u_{t t}-\left(\nabla u^{\top} \operatorname{div} \sigma^{a}-\nabla q, \operatorname{div} \psi^{a}\right) \\
& -\left(\nabla v^{\top} \operatorname{div} \sigma-\nabla p \operatorname{div} \psi\right),
\end{aligned}
$$

since $\nabla(\operatorname{div}(w) \eta+w \cdot \nabla \eta)=\operatorname{div}\left(\nabla w^{\top} \eta+\nabla \eta \otimes w\right)$. Integration by parts yields

$$
\begin{gathered}
\int_{0}^{T} \int_{\Omega^{M}}\left(\nabla w_{t t}\right)^{\top} w^{a}=\int_{0}^{T} \int_{\Omega^{M}}(\nabla w)^{\top} w_{t t}^{a}, \\
\int_{0}^{T} \int_{\Omega^{P}}\left(\nabla u_{t t}\right)^{\top} v=\int_{0}^{T} \int_{\Omega^{P}}(\nabla u)^{\top} v_{t t},
\end{gathered}
$$


and after some arrangements, we obtain

$$
\begin{aligned}
\operatorname{div} \Sigma^{M}= & (\nabla w)^{\top}\left(w_{t t}^{a}-\operatorname{div} S^{a}\right)+\left(\nabla w^{a}\right)^{\top}\left(w_{t t}-\operatorname{div} S\right), \\
\operatorname{div} \Sigma^{P}= & (\nabla u)^{\top}\left(v_{t t}-\operatorname{div} \sigma^{a}\right)+(\nabla v)^{\top}\left(u_{t t}-\operatorname{div} \sigma\right)+\nabla q \operatorname{div} \psi^{a} \\
& +\nabla p \operatorname{div} \psi .
\end{aligned}
$$

Finally, by taking into account the strong systems (2.1) and (5.4), we have the following important results

$$
\operatorname{div} \Sigma^{M}=\operatorname{div} \Sigma^{P}=0 .
$$

In addition, since $V=0$ on $\Gamma_{2}$, and from these last results together with $(5.38,5.39)$, we obtain the final expression for the shape derivative of the functional $\dot{\mathcal{J}}_{\Omega}\left(\varphi_{t}, w\right)$, namely

$$
\dot{\mathcal{J}}_{\Omega}\left(\varphi_{t}, w\right)=\int_{0}^{T} \int_{\Gamma_{1}}\left(\Sigma^{P}-\Sigma^{M}\right) n \cdot V-\int_{0}^{T} \int_{\Gamma_{0}} \Sigma^{P} n \cdot V
$$

with $\Sigma^{M}$ and $\Sigma^{P}$ given respectively by (5.36) and (5.37). The above form of shape derivative of the distributed functional can serve us to identify the shape gradient.

Since the shape functional in question is differentiable in the sense of the shape sensitivity analysis in [12], we can apply the structure theorem to this end. In particular, from the boundary and transmission conditions, namely, (2.5), (5.5) and (2.6), (5.6), respectively, it is straightforward to verify that the above equation holds the structure theorem. Therefore, it is sufficient to take into consideration the speed vector fields normal to the boundaries and the interfaces. This observation influences only two boundary integrals with the Eshelby tensor, and the result is the following.

Corollary 1 The density $\mathfrak{g}$ of the boundary shape gradient of the distributed shape functional is given by the following expression

$$
\langle\mathfrak{g}, V \cdot n\rangle=\int_{0}^{T} \int_{\Gamma_{1}}\left(\left(\Sigma^{P}-\Sigma^{M}\right) n \cdot n\right) V \cdot n-\int_{0}^{T} \int_{\Gamma_{0}}\left(\Sigma^{P} n \cdot n\right) V \cdot n .
$$

As it is indicated before, in order to apply the level-set strategy of shape optimization, it is required that the density $\mathfrak{g}$ of the boundary shape gradient is given by functions supported on the boundaries and on the interfaces.

\section{Conclusions}

In this paper the shape optimization problem for coupled non stationary partial differential equations is analysed. Beside the existence of an optimal shape under realistic conditions, the form of the shape gradient is established in usual expressions necessary for applications of numerical methods, say, for boundary integrals. This means, 
that the shape optimization problem can be solved by the discretization of the continuous shape gradient and the appropriate finite elements in spatial variables and the finite differences in time variable, for example. The numerical realization, however, will be subject to a forthcoming publication.

Open Access This article is distributed under the terms of the Creative Commons Attribution Noncommercial License which permits any noncommercial use, distribution, and reproduction in any medium, provided the original author(s) and source are credited.

\section{Appendix: Proof of Theorem 1}

Without lost of generality, in the proof we can assume that the boundary condition for $q$ (i.e. $q^{P}$ ) as well as the nonhomogeneous terms $f, g$ and $h$ are identically equal to zero. We can use the Galerkin procedure. Thus, we introduce sequences $\left\{\left(\widetilde{\varphi}^{m}, \widetilde{w}^{m}, \widetilde{u}^{m}, \widetilde{q}^{m}\right)_{m \in \mathbb{N}}\right\}$ in $\widetilde{\mathcal{W}}$ and the finite dimensional spaces $\widetilde{\mathcal{W}}^{m}=\operatorname{span}\left\{\left(\widetilde{\varphi}^{1}, \widetilde{w}^{1}, \widetilde{u}^{1}, \widetilde{q}^{1}\right), \ldots,\left(\widetilde{\varphi}^{m}, \widetilde{w}^{m}, \widetilde{u}^{m}, \widetilde{q}^{m}\right)\right\}$ so that the union over all such spaces is dense in $\widetilde{\mathcal{W}}$. Clearly, if we take the test functions $\left(\varphi^{m}, w^{m}, u^{m}, q^{m}\right)$, with $\varphi^{m}=\varphi=\widetilde{\varphi}, w^{m}=w=\widetilde{w}, u^{m}=u=\widetilde{u}$ and $q^{m}=q=\widetilde{q}$, and initial conditions $\varphi^{m}(0)=\varphi_{0}^{m}, \varphi_{t}^{m}(0)=\varphi_{1}^{m}, w^{m}(0)=w_{0}^{m}, w_{t}^{m}(0)=w_{1}^{m}, u^{m}(0)=u_{0}^{m}, u_{t}^{m}(0)=u_{1}^{m}$, such that the sequences $\left(\varphi_{0}^{m}, w_{0}^{m}, u_{0}^{m}, 0\right)$ and $\left(\varphi_{1}^{m}, w_{1}^{m}, u_{1}^{m}, 0\right)$ are convergent in $\tilde{\mathcal{W}}$ and $L^{2}\left(\Omega^{A}\right) \times\left[L^{2}\left(\Omega^{M}\right)\right]^{3} \times\left[L^{2}\left(\Omega^{P}\right)\right]^{3} \times\{0\}$ respectively, then, it follows that problem (3.4) has a local solution in an interval $\left[0, t^{m}\right)$. In order to extend the solution to $[0,+\infty)$, the finite dimensional system of ordinary differential equations associated to (3.4) now reads as follows. For any $(\widetilde{\varphi}, \widetilde{w}, \widetilde{u}, \widetilde{q}) \in \tilde{\mathcal{W}}^{m}$,

$$
\left\{\begin{array}{l}
\frac{1}{c^{2}}\left\langle\varphi_{t t}^{m}(t), \widetilde{\varphi}\right\rangle_{\Omega^{A}}+a_{A}\left(\varphi^{m}(t), \widetilde{\varphi}\right)-\left\langle w_{t}^{m}(t) \cdot n, \widetilde{\varphi}\right\rangle_{\Gamma_{2}}+\frac{1}{c}\left\langle\varphi_{t}^{m}, \widetilde{\varphi}\right\rangle_{\Gamma_{3}} \\
\quad+\left\langle w_{t t}^{m}(t), \widetilde{w}\right\rangle_{\Omega^{M}}+a_{M}\left(w^{m}(t), \widetilde{w}\right)+\left\langle\varphi_{t}^{m}(t), \widetilde{w} \cdot n\right\rangle_{\Gamma_{2}} \\
\quad+\left\langle u_{t t}^{m}(t), \widetilde{u}\right\rangle_{\Omega^{P}}+a_{M M}\left(u^{m}(t), \widetilde{u}\right)+a_{E M}\left(q^{m}(t), \widetilde{u}\right) \\
\quad+a_{E E}\left(q^{m}(t), \widetilde{q}\right)-a_{M E}\left(u^{m}(t), \widetilde{q}\right)=0 .
\end{array}\right.
$$

Taking as test functions $\left(\varphi_{t}^{m}(t), w_{t}^{m}(t), 0,0\right)$ in (A.1) and integrating over $[0, t]$ we obtain

$$
\begin{aligned}
\frac{1}{2 c^{2}}\left\|\varphi_{t}^{m}\right\|^{2}+\frac{1}{2}\left\|w_{t}^{m}\right\|^{2}+\frac{1}{2} a_{A}\left(\varphi^{m}(t), \varphi^{m}(t)\right)+\frac{1}{2} a_{M}\left(w^{m}(t), w^{m}(t)\right) \\
\quad+\frac{1}{c} \int_{0}^{t}\left\|\varphi_{s}^{m}\right\|_{L^{3}\left(\Gamma_{2}\right)}^{2} d s \\
=\frac{1}{2 c^{2}}\left\|\varphi_{t}^{m}(0)\right\|^{2}+\frac{1}{2}\left\|w_{t}^{m}(0)\right\|^{2}+\frac{1}{2} a_{A}\left(\varphi^{m}(0), \varphi^{m}(0)\right) \\
\quad+\frac{1}{2} a_{M}\left(w^{m}(0), w^{m}(0)\right) .
\end{aligned}
$$


Now as test functions in (A.1) we take $\left(0,0, u_{t}^{m}(t), 0\right),\left(0,0,0, q_{t}^{m}(t)\right)$ and $\left(0,0,0, q^{m}(t)\right)$ and to obtain the identities

$$
\frac{1}{2} \frac{d}{d t}\left\{\left\|u_{t}^{m}\right\|^{2}+a_{M M}\left(u^{m}, u^{m}\right)\right\}+a_{E M}\left(q^{m}, u_{t}^{m}\right)=0,
$$

and

$$
a_{E E}\left(q^{m}, q_{t}^{m}\right)=a_{M E}\left(u^{m}, q_{t}^{m}\right) \quad \text { and } \quad a_{E E}\left(q^{m}, q^{m}\right)=a_{M E}\left(u^{m}, q^{m}\right),
$$

respectively. Then, it follows that

$$
\begin{aligned}
\frac{d}{d t} a_{E E}\left(q^{m}, q^{m}\right) & =a_{M E}\left(u_{t}^{m}, q^{m}\right)+a_{M E}\left(u^{m}, q_{t}^{m}\right) \\
& =a_{M E}\left(u_{t}^{m}, q^{m}\right)+a_{E E}\left(q^{m}, q_{t}^{m}\right) .
\end{aligned}
$$

Therefore

$$
\frac{1}{2} \frac{d}{d t} a_{E E}\left(q^{m}, q^{m}\right)=a_{M E}\left(u_{t}^{m}, q^{m}\right)=a_{E M}\left(q^{m}, u_{t}^{m}\right),
$$

by symmetry. Using (A.4) in (A.3) and integrating over $[0, t]$ we obtain

$$
\begin{aligned}
& \left\|u_{t}^{m}\right\|^{2}+a_{M M}\left(u^{m}, u^{m}\right)+a_{E E}\left(q^{m}, q^{m}\right) \\
& \quad=\left\|u_{1}^{m}\right\|^{2}+a_{M M}\left(u_{0}^{m}, u_{0}^{m}\right)+a_{E E}\left(q^{m}(0), q^{m}(0)\right) .
\end{aligned}
$$

In order to obtain an initial condition for $q^{m}$ we need to solve

$$
a_{E E}\left(q^{m}(0), \xi\right)=a_{M E}\left(u_{0}^{m}, \xi\right),
$$

for any $\xi \in\left\{\operatorname{span}\left\{\xi^{1}, \xi^{2}, \ldots, \xi^{m}\right\}, \xi^{i} \in H^{1}(\Omega): \xi^{i}=0\right.$ on $\left.\Gamma_{1}\right\}$. Since we know the regularity of $u_{0}^{m}$ we can apply the Lax-Milgran lemma and obtain a unique solution $q^{m}(0)$ belonging to $H^{1}(\Omega)$ with $q^{m}(x, 0)=0$ on $\Gamma_{1}$. Furthermore

$$
\left\|q^{m}(0)\right\| \leq c\left\|u_{0}^{m}\right\|_{\left[H^{1}\left(\Omega^{P}\right)\right]^{3}} .
$$

Using the coercivity of the bilinear forms $a_{M M}$ and $a_{E E}$ in $\mathcal{W}_{P}$ and $\mathcal{W}_{E}$ respectively to obtain from (A.3) and (A.7). In case we consider $f, g, h$ and $q^{D}$ different from zero, we use Gronwall's inequality at this point.

$$
\left\|u_{t}^{m}\right\|_{\left[L^{2}\left(\Omega^{P}\right)\right]^{3}}^{2}+\left\|u^{m}\right\|_{\mathcal{W}_{P}}^{2}+\left\|q^{m}\right\|_{\mathcal{W}_{E}}^{2} \leq C\left\{\left\|u_{1}^{m}\right\|_{\left[L^{2}\left(\Omega^{P}\right)\right]^{3}}^{2}+\left\|u_{0}^{m}\right\|_{\left[\mathcal{W}_{P}\right.}^{2}\right\},
$$

for some positive constant $C$. A standard argument shows that also

$$
\left\|\varphi_{t t}^{m}\right\|^{*},\left\|w_{t t}^{m}\right\|^{*},\left\|u_{t t}^{m}\right\|^{*} \leq C .
$$

Using the a priori energy estimates (A.10) and (A.11) we can then extract subsequences $\left\{\varphi^{m}\right\},\left\{\varphi_{t}^{m}\right\},\left\{\varphi_{t t}^{m}\right\} ;\left\{w^{m}\right\},\left\{w_{t}^{m}\right\},\left\{w_{t t}^{m}\right\} ;\left\{u^{m}\right\},\left\{u_{t}^{m}\right\},\left\{u_{t t}^{m}\right\}$, which we relabel by original indices converging for $K:=A, M, P$ weak- $(\star)$ in $L^{\infty}\left(0, T ; \mathcal{W}_{K}(\Omega)\right)$, and weak in $L^{2}\left(0, T ; \mathcal{W}_{K}(\Omega)^{*}\right)$, respectively, to elements $\varphi^{*}, \varphi_{t}^{*}, \varphi_{t t}^{*} ; w^{*}, w_{t}^{*}, w_{t t}^{*}$; 
$u^{*}, u_{t}^{*}, u_{t t}^{*}$. Standard arguments reveal that these elements solve the weak system (3.4) and that the initial data are matched in the corresponding spaces as well.

As for the second part of the theorem, we first differentiate the approximate weak system and take $\left(\varphi_{t t}^{m}(t), 0,0,0\right)$ and then $\left(0, w_{t t}^{m}(t), 0,0\right)$. One adds the results and integrates with respect to time to obtain

$$
\begin{aligned}
\frac{1}{c^{2}}\left\|\varphi_{t t}^{m}(t)\right\|^{2}+\left\|w_{t t}^{m}(t)\right\|^{2}+a_{A}\left(\varphi_{t}^{m}(t), \varphi_{t}^{m}(t)\right)+a_{M}\left(w_{t}^{m}(t), w_{t}^{m}(t)\right) \\
\quad+\frac{2}{c} \int_{0}^{t}\left|\varphi_{t t}^{m}(t)\right|_{\Gamma_{3}}^{2} d \gamma \\
\leq\left\{\frac{1}{c^{2}}\left\|\varphi_{t t}^{m}(0)\right\|^{2}+\left\|w_{t t}^{m}(0)\right\|^{2}+a_{\Omega^{M}}\left(w_{t}^{m}(0), w_{t}^{m}(0)\right)+a_{A}\left(\varphi_{t}^{m}(0), \varphi_{t}^{m}(0)\right)\right\} .
\end{aligned}
$$

As for the piezoelectric part, we take $\left(0,0, u_{t t}^{m}(t), 0\right)$ as test functions and then, after another differentiation of the weak form with respect to $t,\left(0,0,0, q_{t}^{m}(t)\right.$. We obtain

$$
\frac{1}{2} \frac{d}{d t}\left\{\left\|u_{t t}\right\|^{2}+a_{\Omega^{P}}^{M M}\left(u_{t}^{m}(t), u_{t}^{m}(t)\right)+a_{\Omega^{P}}^{E E}\left(q_{t}^{m}(t), q_{t}^{m}(t)\right)\right\}=0 .
$$

Integration with respect to time leads to:

$$
\begin{aligned}
& \left\|u_{t t}^{m}(t)\right\|^{2}+\left\|u_{t}^{m}(t)\right\|_{\mathcal{W}_{P}}^{2}+\left\|q_{t}^{m}(t)\right\|_{\mathcal{W}}^{2} \mathcal{W}_{E} \\
& \quad \leq C\left\{\left\|u_{t t}^{m}(0)\right\|^{2}+\left\|u_{t}^{m}(0)\right\|_{\mathcal{W}_{P}}^{2}+\left\|q_{t}^{m}(0)\right\|_{\mathcal{W}_{E}}^{2}\right\} .
\end{aligned}
$$

We need estimates on $\left\|\varphi_{t t}^{m}(0)\right\|,\left\|w_{t t}^{m}(0)\right\|_{\left[L^{2}\left(\Omega^{M}\right)\right]^{3}},\left\|u_{t t}^{m}(0)\right\|_{\left[L^{2}\left(\Omega^{P}\right)\right]^{3}}$ and $\left\|q_{t}^{m}(0)\right\|_{\mathcal{W}}$ in terms of our data. As now $u_{t}^{m}(0) \in \mathcal{W}_{P}$ we can uniquely solve the second equation of (3.4) to obtain

$$
\left\|q_{t}^{m}(0)\right\| \mathcal{W}_{E} \leq C\left\{\left\|u_{t}^{m}(0)\right\| \mathcal{W}_{P}\right\} .
$$

Moreover, for given $u^{m}(0) \in H^{2}\left(\Omega^{P}\right)$ let $q^{m}(0) \in H^{2}\left(\Omega^{P}\right)$ be such that

$$
\begin{cases}\operatorname{div}(D \nabla q)=\operatorname{div}\left(P^{\top} \nabla^{s} u^{m}(0)\right) & \text { in } \Omega^{P} \\ D \nabla q \cdot n=P^{\top} \nabla^{s} u \cdot n & \text { on } \Gamma_{1} \\ q=0 & \text { on } \Gamma_{0} .\end{cases}
$$

Then $\left\|q^{m}(0)\right\|_{H^{2}(\Omega)^{P}} \leq\left\{\left\|u^{m}(0)\right\|_{H^{2}\left(\Omega^{P}\right)^{3}}\right\}$ and after evaluating the strong solution at $t=0$ we obtain

$$
\left\|u_{t t}^{m}(0)\right\| \leq C\left\|u^{m}(0)\right\|_{H^{2}\left(\Omega^{P}\right)^{3}} .
$$

We can now proceed as before, in order to obtain the a priori estimate

$$
\begin{aligned}
& \left\|u_{t t}^{m}(t)\right\|_{H^{2}\left(\Omega^{P}\right)^{3}}^{2}+\left\|u_{t}^{m}(t)\right\|_{\mathcal{W}}^{2}+\left\|u_{t t}^{m}(t)\right\|_{\left[L^{2}\left(\Omega^{P}\right)\right]^{3}}^{2}+\left\|q^{m}(t)\right\|_{H^{2}\left(\Omega^{P}\right)}^{2}+\left\|q_{t}^{m}(t)\right\|_{\mathcal{W}}^{2} \\
& \leq C\left\{\left\|u^{m}(0)\right\|_{H^{2}\left(\Omega^{P}\right)^{3}}^{2}+\left\|u_{t}^{m}(0)\right\|_{\mathcal{W}}^{2}\right\} .
\end{aligned}
$$


We then subtract weak- $(\star)$ convergent subsequences and pass to the limit in the equations. The fulfillment of the initial data is proved by a standard argument. Note that also non-homogenous boundary conditions for $q$ (and $u$ ) can be easily handled.

\section{References}

1. Cagnol, J., Zolésio, J.P.: Shape derivative in the wave equation with Dirichlet boundary conditions. J. Differ. Equ. 334(3), 175-210 (1999)

2. Cardone, G., Nazarov, S.A., Sokołowski, J.: Asymptotic analysis, polarization matrices, and topological derivatives for piezoelectric materials with small voids. SIAM J. Control Optim. 48(6), 3925-3961 (2010)

3. Delfour, M.C., Zolésio, J.P.: Shapes and Geometries. Advances in Design and Control. SIAM, Philadelphia (2001)

4. Eshelby, J.D.: The elastic energy-momentum tensor. J. Elast. 5(3-4), 321-335 (1975)

5. Geis, W., Mishuris, G., Sändig, A.-M.: Piezoelectricity in multi-layer actuators modelling and analysis in two and three dimensions. Berichte aus dem Institut für Angewandte Analysis und Numerische Simulation 23, Universität Stuttgart (2003)

6. Gurtin, M.E.: An Introduction to Continuum Mechanics. Mathematics in Science and Engineering, vol. 158. Academic Press, New York (1981)

7. Gurtin, M.E.: Configurational Forces as Basic Concept of Continuum Physics. Applied Mathematical Sciences, vol. 137. Springer, New York (2000)

8. Lasiecka, I.: Mathematical Control Theory of Coupled PDEs. CBMS-NSF Regional Conference Series in Applied Mathematics, vol. 75. SIAM, Philadelphia (2002)

9. Leugering, G., Novotny, A.A., Menzala, G. Perla, Sokołowski, J.: Shape sensitivity analysis of a quasi-electrostatic piezoelectric system in multilayered media. Math. Methods Appl. Sci. 33(17), 2118-2131 (2010)

10. Melnik, V.N.: Existence and uniqueness theorems of the generalized solution for a class of nonstationary problem of coupled electroelasticity. Sov. Math., Izv. VUZ, Math. 35(4), 24-32 (1991)

11. Mercier, D., Nicaise, S.: Existence, uniqueness and regularity results for piezoelectric systems. SIAM J. Math. Anal. 37, 651-672 (2005)

12. Sokołowski, J., Zolésio, J.P.: Introduction to Shape Optimization-Shape Sensitivity Analysis. Springer, New York (1992)

13. Wein, F., Kaltenbacher, M., Schury, F., Leugering, G., Bänsch, E.: Topology optimization of piezoelectric actuators using the simp method. In: 10th Workshop on Optimization and Inverse Problems in Electromagnetism-OIPE, Ilmenau, Germany (2008)

14. Wein, F., Kaltenbacher, M., Schury, F., Leugering, G., Bänsch, E.: Topology optimization of a piezoelectric loudspeaker coupled with the acoustic domain. In: Proceedings WCSMO-08, Lisbon, Portugal (2009)

15. Wein, F., Kaltenbacher, M., Schury, F., Leugering, G., Bänsch, E.: Topology optimization of a piezoelectric-mechanical actuator withsingle- and multiple-frequency excitation. Int. J. Appl. Electromagn. Mech. 30(3-4), 201-221 (2009)

16. Wein, F., Kaltenbacher, M., Kaltenbacher, B., Leugering, G., Bänsch, E., Schury, F.: On the effect of self-penalization of piezoelectric composites in topology optimization. Struct. Multidiscip. Optim. 43, 405-417 (2011) 